\title{
30. LOW PRESSURE EXPERIMENTAL CONSTRAINTS ON THE EVOLUTION OF BASALTIC LAVAS FROM SITE 917, SOUTHEAST GREENLAND CONTINENTAL MARGIN ${ }^{1}$
}

\author{
P. Thy, ${ }^{2}$ C.E. Lesher, ${ }^{2}$ and M.S. Fram ${ }^{2}$
}

\begin{abstract}
One-atmosphere melting experiments conducted on lavas recovered from Site 917 on the southeast Greenland Margin define the liquid line of descent for primitive basalts for the continental volcanic succession. The most primitive aphyric lava from the Lower Series, with $9.1 \mathrm{wt} \% \mathrm{MgO}$, defines the crystallization sequence of olivine $\left(1247 \pm 5^{\circ} \mathrm{C}\right)$, plagioclase $(1219 \pm$ $\left.5^{\circ} \mathrm{C}\right)$, followed by augite $\left(1182 \pm 5^{\circ} \mathrm{C}\right)$. A sample from the Upper Series with $10.7 \mathrm{wt} \% \mathrm{MgO}$ shows a similar crystallization order with plagioclase and olivine appearing at $1228 \pm 5^{\circ} \mathrm{C}$ and augite crystallizing at $1182 \pm 5^{\circ} \mathrm{C}$. The liquid lines of descent are in close agreement with the compositional relationships for the primitive members of the Lower and Upper Series and the oceanic succession drilled at Sites 915 and 918, but contrast sharply with those of differentiated lavas from the Middle Series of Site 917 modified by crustal contamination. Some differentiated lavas from the Lower Series indicate excessive olivine fractionation due to suppressed plagioclase crystallization at elevated $\mathrm{f}_{\mathrm{H} 2 \mathrm{O}}$. Consideration of $\mathrm{Fe}-\mathrm{Mg}$ exchange equilibrium for high$\mathrm{MgO}$ aphyric lavas and picrites recovered at Site 917 confirms the accumulative nature of the picrites and is used to calculate melts in equilibrium with typical mantle source. We show that major element compositions for these calculated primary melts $(15-18 \mathrm{wt} \% \mathrm{MgO})$ are consistent with previous estimates for rifted margin basalts in the North Atlantic. The production of more $\mathrm{Fe}$ - and Ti-rich primary melts with time suggests that the efficiency of melt segregation and potential temperature increased during production of the volcanic succession at Site 917.
\end{abstract}

\section{INTRODUCTION}

Ocean Drilling Program Leg 152 explored the early Tertiary tectonic and magmatic rifting history of the North Atlantic continental margin and the initial transition of the margin into an ocean basin. Magmatic activity during rifting and transition to spreading is recorded by extensive development of seaward-dipping reflectors and continental flood basalts along the East Greenland coast. The succession collected at Site 917 represents the oldest drilled portion of the seaward-dipping reflector series and is composed of three well-defined stratigraphic units: an Upper Series of primitive lavas, a Middle Series of evolved lavas, and a Lower Series including both primitive and evolved lavas (Larsen, Saunders, Clift, et al., 1994; Fitton et al., Chap. 28, this volume). The sampled lavas range in composition from picrite to dacite and are geochemically distinct from the Fe-Ti basalts common to most other early Tertiary volcanic successions on the North Atlantic margins (Larsen et al., Chap. 27, this volume). The upper part at Site 917, as well as the magmatic units collected at Sites 915 and 918 , record the transition into ocean spreading (Fitton et al., Chap. 28, this volume; Fram et al., this volume).

The present study was performed with the purpose of exploring phase equilibrium constraints on magmatic evolution during the construction of the seaward-dipping reflector series and transition to oceanic volcanism. With this in mind, this paper presents initial results of low pressure melting experiments on basaltic lavas from the Lower and Upper Series at Site 917. The experimental results define the low pressure melting relations and liquid lines of descent. These results provide constraints on the magmatic evolution of the volcanic succession and illustrate differences between closed system evolution and open system behavior involving replenishment and crustal contamination. We suggest that the mafic lavas from the Lower Series experienced protracted olivine fractionation due to an expansion of the olivine primary phase volume with modest contamination and hydration by interaction with continental crust. This accounts for the

${ }^{1}$ Saunders, A.D., Larsen, H.C., and Wise, S.W., Jr. (Eds.), 1998. Proc. ODP, Sci. Results, 152: College Station, TX (Ocean Drilling Program).

${ }^{2}$ Department of Geology, University of California, Davis, CA 95616, U.S.A. thy@geology.ucdavis.edu high normative plagioclase content of certain evolved aphyric lavas. Lavas from the Upper Series at Site 917, as well as from Sites 915 and 918 , were governed primarily by fractionation and magma replenishment in low pressure magma chambers. We confirm the olivine accumulative origin of most picrites recovered from both the Lower and Upper Series and evaluate the major element composition of primary mantle-derived magmas for the continental volcanic succession.

\section{SAMPLES}

For this experimental study we selected two representative samples: one belonging to flow Unit 13 in the Upper Series (Sample 152917A-11R-4, 26-30 cm, Piece 1B) and one from flow Unit 84 in the Lower Series (Sample 152-917A-86R-7, 45-49 cm, Piece 1). These samples are designated as 11R4 and 86R7, respectively, in the following discussions. They are among the most magnesian aphyric lavas recovered from Site 917 (10.7 and $9.1 \mathrm{wt} \% \mathrm{MgO}$, respectively; Table 1) and are considered representative magmatic liquid compositions. Although many of the olivine phyric and picritic lavas are more magnesian, their phyric nature precludes unambiguous designation as liquid compositions.

The Lower Series sample (86R7) consists of a groundmass of olivine, plagioclase, augite, and Fe-Ti oxide minerals. The composition of olivine ranges from $\mathrm{Fo}_{65}$ to $\mathrm{Fo}_{60}$, while plagioclase is typically $\mathrm{An}_{60}$, but compositions up to $\mathrm{An}_{68}$ are present. Augite shows a limited compositional range of $\mathrm{En}_{48-44} \mathrm{Fs}_{11-16} \mathrm{Wo}_{41-39}$ and $\mathrm{Ti} / \mathrm{Al}$ ratios of about 0.22 . Both samples have $\mathrm{Mg} /\left(\mathrm{Mg}+\mathrm{Fe}_{\text {total }}\right)$ ratios of 0.63 , but they differ in the proportions of normative hypersthene and olivine, as well as in the concentrations of trace elements (Table 1). Sample 86R7 has lower concentrations of $\mathrm{Ni}, \mathrm{Cr}$, and $\mathrm{V}$ and almost three times more $\mathrm{Sr}$ than 11R4. The samples are slightly altered and hydrated to clay minerals and zeolites resulting in loss-on-ignition of 2-3 wt $\%$ and ferric/ferrous iron ratios near unity (Table 1).

\section{EXPERIMENTAL AND ANALYTICAL TECHNIQUES}

The two rock samples were ground in a tungsten-carbide shatterbox for $2 \mathrm{~min}$ and subsequently in an agate mortar under acetone to 
Table 1. Composition of starting material and analytical precision.

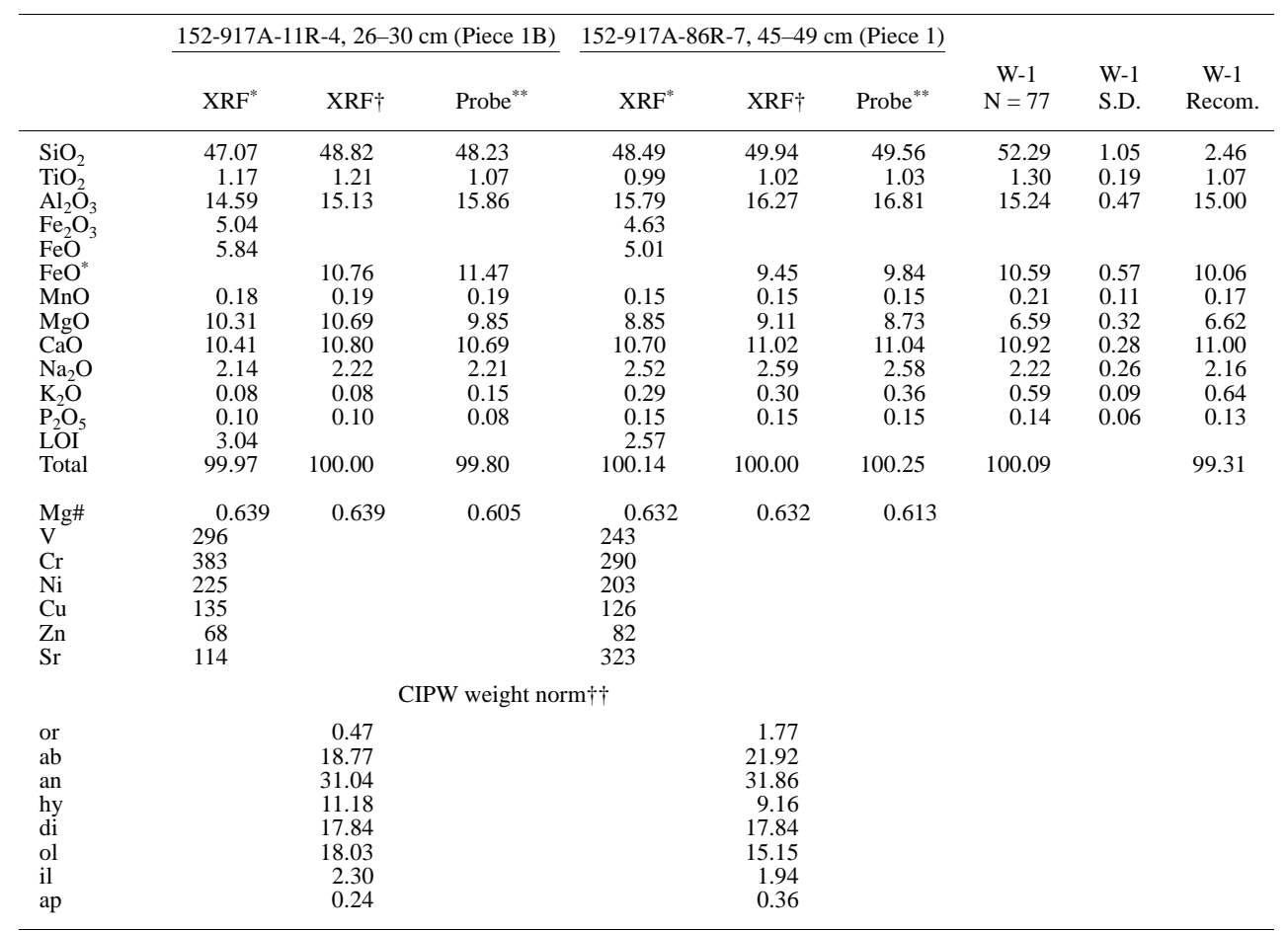

Notes: ${ }^{*}=$ from Larsen et al. (Chap. 27, this volume) determined by XRF. LOI is loss of ignition. $\dagger=$ XRF analysis calculated anhydrous with all iron as FeO. ${ }^{* *}=$ electron microprobe analysis of superliquidus glass. $\dagger \dagger=\mathrm{CIPW}$ weight norm calculated with all iron as $\mathrm{FeO}^{*}$. FeO* is total iron as $\mathrm{FeO}$. $\mathrm{Mg} \#$ is $\mathrm{Mg} /(\mathrm{Mg}+\mathrm{Fe})$ ratio with all iron as Fe ${ }^{2+}$. W-1 is international reference sample W-1 fused to a glass. First column is the average of 77 point microprobe analyses, second column is one standard deviation, third column is compiled and recommended value from Govindaraju (1989).

an estimated grain size of $\leq 10 \mu \mathrm{m}$. The powders were dried for $24 \mathrm{hr}$ in a vacuum oven at $110^{\circ} \mathrm{C}$ and then stored in a desiccator. About 0.5 $\mathrm{g}$ of powder for each sample was mixed with polyvinyl alcohol and pressed into pellets and dried. These pellets were broken into $\sim 50 \mathrm{mg}$ pieces and fused to 0.004-in Fe-Pt alloy suspension wire. The wire was prepared by iron electroplating and annealing as discussed by Grove (1981). The experimental charges were suspended in a vertical quench furnace at the desired run temperatures and 1 atm pressure, and quenched in air. Temperature was monitored by a Pt/90Pt-10Rh thermocouple calibrated against the melting point of gold (Biggar, 1972). The furnace atmosphere was controlled to the fayalite-magnetite-quartz (FMQ) oxygen buffer using a $\mathrm{CO}-\mathrm{CO}_{2}$ gas mixture and monitored by a solid $\mathrm{ZrO}_{2}$-ceramic oxygen probe calibrated against the Ni-NiO reaction. To minimize sodium loss by volatilization, the total gas flow rate was maintained at $\sim 0.01 \mathrm{~cm}^{2} \mathrm{~s}^{-1}$. Run duration ranged from $19 \mathrm{hr}$ at near liquidus conditions to $90 \mathrm{hr}$ at near solidus conditions. Table 2 provides a summary of experimental conditions.

The experimental run products were examined by reflected light and scanning electron microscopy, and the phase compositions were determined using the Cameca SX50 electron microprobe. The microprobe was operated with an accelerating voltage of $15 \mathrm{kV}$, a beam current of $10 \mathrm{nA}$, and count times of 10-30 s. All phases were analyzed using natural minerals as standards. Very small and very large grains were avoided, restricting the grain-size population analyzed to those most likely to have grown in equilibrium during the experiments $(100-20 \mu \mathrm{m})$. Grains showing unreacted portions or patchy zoning on the backscattered electron images were avoided or only analyzed on their homogeneous rims. A beam diameter of $1 \mu \mathrm{m}$ was used for minerals, while glasses were normally analyzed with a 10 $\mu \mathrm{m}$ broad beam to minimize volatilization of sodium. Some low temperature glasses were analyzed with a narrow beam because a small glass area prohibited the use of a broad beam. An internal glass standard prepared from international rock standard W-1 (Govindaraju,
1989) was analyzed concurrently and used to evaluate analytical precision and accuracy (Table 1). The resulting analyses were screened by compositional and stoichiometric criteria and are considered representative for the experiments. The average glass and mineral compositions reported in Tables 3-6 represent averages of 3-13 individual analyses of each phase of a given run. The redox state of iron in liquids at the experimental conditions was estimated using the equations by Kilinc et al. (1983).

The standard deviations of the replicate analyses reported in Tables 3-6 reflect the homogeneity of the phases. The standard deviations for the experimental glass (Table 2) are comparable to the W-1 standard (Table 1). Likewise, olivines show a high degree of homogeneity (Table 4), while larger standard deviations are noted for the plagioclase (Table 5) and augite (Table 6) analyses. In general, the degree of heterogeneity is greater for the lower temperature runs and likely reflects incomplete reaction of the starting material. For example, individual plagioclase compositions for Run $86 \mathrm{R} 7-8$ (at $1111^{\circ} \mathrm{C}$ ) range from $\mathrm{An}_{53}$ to $\mathrm{An}_{65}$. The more calcic plagioclase most likely represents fragments of unequilibrated plagioclase $\left(\mathrm{An}_{60-68}\right)$ of the starting material. Such problems are common at low temperatures in melting studies of this kind and need to be considered when interpreting results of the lower temperature runs.

The modal proportions of phases in the experimental charges were determined by weighted mass balance using the compositions of the phases as determined by electron microprobe (Bryan et al., 1969). All of the oxides were assigned a weighting factor of 1.0, except $\mathrm{SiO}_{2}$ and $\mathrm{Al}_{2} \mathrm{O}_{3}$, which were weighted by 0.4 and 0.5 , respectively. The results are presented in Table 2 . Losses of sodium to the furnace gas and under the electron microbeam during analysis were estimated by including $\mathrm{Na}_{2} \mathrm{O}$ as a phase in the mass balance calculation. Table 2 shows that less than $10 \%$ by weight of $\mathrm{Na}_{2} \mathrm{O}$ is unaccounted for in a given charge. The loss of sodium is corrected for in the reported glass analyses in Table 3. The low sum of the squares of the 
Table 2. Experimental conditions and results.

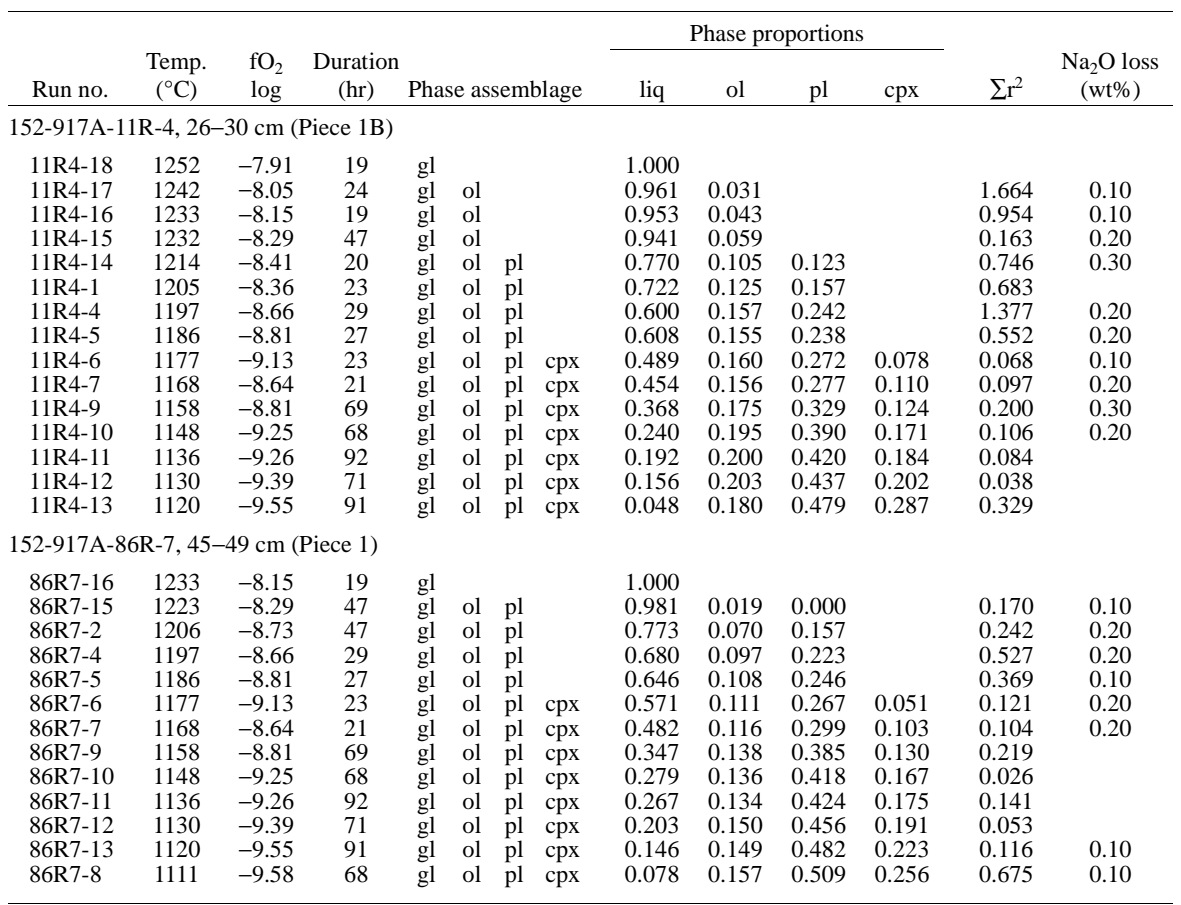

Notes: Abbreviations of experimental phase assemblage are $\mathrm{gl}=\mathrm{glass} ; \mathrm{ol}=$ olivine; $\mathrm{pl}=$ plagioclase; $\mathrm{cpx}=$ augite. The phase proportions $($ wt $\%$ ) are calculated by least squares mixing calculations, as explained in the text. $\sum \mathrm{r}^{2}$ is the sum of squares of residuals. The loss of $\mathrm{Na}_{2} \mathrm{O}$ has been estimated by including $\mathrm{Na}_{2} \mathrm{O}$ as a phase in the mixing calculations.

residuals for the least-squares fit of the analyzed charge to the original bulk composition (Table 2) further shows that iron gain or loss to the alloyed suspension loop was negligible.

\section{EXPERIMENTAL RESULTS}

\section{Phase Appearance and Proportions}

The melting sequences and temperature-composition relations are summarized in Table 2 and Figure 1. Sample 11R4 shows the following sequence of liquidus phases: olivine appearing at $1247 \pm 5^{\circ} \mathrm{C}$, plagioclase at $1219 \pm 5^{\circ} \mathrm{C}$, and augite at $1182 \pm 5^{\circ} \mathrm{C}$. Sample $86 \mathrm{R} 7$ crystallizes the same sequence of phases at nearly the same temperatures, except that the liquidus temperature for olivine is displaced to a slightly lower temperature $\left(1228 \pm 5^{\circ} \mathrm{C}\right)$, and the appearance temperature of plagioclase is slightly higher $\left(1228 \pm 5^{\circ} \mathrm{C}\right)$. These relations show that primitive basaltic magmas from Site 917 crystallizing under low pressure conditions will be saturated with olivine above about $1225^{\circ} \mathrm{C}$, olivine and plagioclase between $1225^{\circ} \mathrm{C}$ and $1180^{\circ} \mathrm{C}$, and olivine, plagioclase, and augite below $1180^{\circ} \mathrm{C}$. The variation in temperature with $\mathrm{MgO}$ content ( $\mathrm{wt} \%$ ) of all experimental liquids given in Table 3 yields the relation: $\mathrm{T}\left( \pm 5^{\circ} \mathrm{C}\right)=24 \cdot \mathrm{MgO}+1022$ (Fig. 2). This relationship provides an estimate of minimum eruption temperature for aphyric lavas with $\mathrm{MgO}$ contents within the range of the experiments. Low-Ca pyroxene is not observed in the experiments, although it may be stable below $1111^{\circ} \mathrm{C}$. This is consistent with the absence of low-Ca pyroxene as either a phenocryst or groundmass phase in Site 917 lavas (Larsen, Saunders, Clift, et al., 1994). Likewise, chromium spinel may be present in a few of the higher temperature runs, but its low abundance and extremely small size prohibits detailed evaluation.

The mass balance calculations show that the experiments characterize the phase relations over more than $90 \%$ of the crystallization interval. It is also noteworthy that at least one-half of the experiments performed for each bulk composition are multisaturated with olivine, plagioclase, and augite. Neither sample shows a significant inflection with the appearance of augite in plots of temperature or composition vs. liquid fraction (Fig. 1).

\section{Solid Phase Chemistry and Mineral-Liquid Relations}

As noted, olivine is the liquidus phase for both samples and is present throughout the investigated melting interval. Olivine compositions range from $\mathrm{Fo}_{85}$ to $\mathrm{Fo}_{61}$ and, as expected, correlate with melting temperature and liquid $\mathrm{Mg} /\left(\mathrm{Mg}+\mathrm{Fe}^{2+}\right)$ ratios $(\mathrm{Mg} \#)$. Using $\mathrm{Fe}^{3+}$ $\mathrm{Fe}^{2+}$ ratios for the experimental liquids derived from the model of Kilinc et al. (1983) (i.e., $0.079 \pm 0.004$ for $11 \mathrm{R} 4$ and $0.081 \pm 0.003$ for 86R7), the distribution coefficient for $\mathrm{Mg}$ and $\mathrm{Fe}^{2+}$ between oliv-

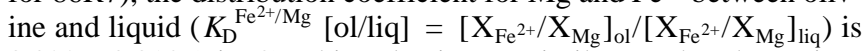
$0.322 \pm 0.018$ (Fig. 3). This value is very similar to other determinations for basaltic compositions (Roeder and Emslie, 1970; Ulmer, 1989) and demonstrates that olivine and liquid are well equilibrated in all runs.

Plagioclases range in composition from $\mathrm{An}_{79}$ to $\mathrm{An}_{60}$. The relationship between plagioclase and liquid compositions can be expressed in terms of the exchange of anorthite (An) and albite (Ab) components, $K_{\mathrm{D}}=(\mathrm{Ab} / \mathrm{An})_{\mathrm{pl}} /(\mathrm{Ab} / \mathrm{An})_{\text {liq }}$ (Weaver and Langmuir, 1990). The $K_{\mathrm{D}}$ values computed in this way range from 0.38 to 0.62 with a general trend toward higher values for more albitic liquids at lower temperature. The errors associated with these $K_{\mathrm{D}} \mathrm{s}$, however, are especially large for the low temperature runs because of the variability in plagioclase compositions due to the incomplete equilibration between plagioclase and liquid noted earlier.

Augite composition varies from $\mathrm{En}_{47} \mathrm{Fs}_{11} \mathrm{Wo}_{42}$ to $\mathrm{En}_{44} \mathrm{Fs}_{18} \mathrm{Wo}_{37}$. The quadrilateral components of the augites show systematic and nearly identical reduction of enstatite and wollastonite components with decreasing melting temperature (Table 6). There is also a marked difference in nonquadrilateral components for the two groups of augites. The 11R4 augites have systematically higher Al contents as compared with the 86R7 augites, resulting in Ti/Al ratios of about 0.15 for $11 \mathrm{R} 4$ augites and between 0.15 and 0.30 for $86 \mathrm{R} 7$ augites. If ferrous iron is estimated by charge balancing cation substitutions 


\begin{tabular}{|c|c|c|c|c|c|c|c|c|c|c|c|c|c|c|c|c|c|c|c|c|c|c|c|c|c|c|}
\hline \multirow[b]{2}{*}{ Run no. } & \multirow[b]{2}{*}{$\begin{array}{l}\text { Temp. } \\
\left({ }^{\circ} \mathrm{C}\right)\end{array}$} & \multirow[b]{2}{*}{$\mathrm{N}$} & \multirow[b]{2}{*}{$\mathrm{SiO}_{2}$} & \multirow[b]{2}{*}{$\mathrm{TiO}_{2}$} & \multirow[b]{2}{*}{$\mathrm{Al}_{2} \mathrm{O}_{3}$} & \multirow[b]{2}{*}{$\mathrm{FeO}^{*}$} & \multirow[b]{2}{*}{$\mathrm{MnO}$} & \multirow[b]{2}{*}{$\mathrm{MgO}$} & \multirow[b]{2}{*}{$\mathrm{CaO}$} & \multirow[b]{2}{*}{$\mathrm{Na}_{2} \mathrm{O}$} & \multirow[b]{2}{*}{$\mathrm{K}_{2} \mathrm{O}$} & \multirow[b]{2}{*}{$\mathrm{P}_{2} \mathrm{O}_{5}$} & \multirow[b]{2}{*}{$\mathrm{Cr}_{2} \mathrm{O}_{3}$} & \multirow[b]{2}{*}{$\begin{array}{l}\mathrm{Na}_{2} \mathrm{O} \\
\text { corr. }\end{array}$} & \multirow[b]{2}{*}{ Mg\# } & \multicolumn{11}{|c|}{1 standard deviation } \\
\hline & & & & & & & & & & & & & & & & $\mathrm{SiO}_{2}$ & $\mathrm{TiO}_{2}$ & $\mathrm{Al}_{2} \mathrm{O}_{3}$ & $\mathrm{FeO}$ & $\mathrm{MnO}$ & $\mathrm{MgO}$ & $\mathrm{CaO}$ & $\mathrm{Na}_{2} \mathrm{O}$ & $\mathrm{K}_{2} \mathrm{O}$ & $\mathrm{P}_{2} \mathrm{O}_{5}$ & $\mathrm{Cr}_{2} \mathrm{O}_{3}$ \\
\hline \multicolumn{27}{|c|}{ 152-917A-11R-4, 26-30 cm (Piece 1B) } \\
\hline 11R4-18 & 1252 & 11 & 48.23 & 1.07 & 15.86 & 11.47 & 0.19 & 9.85 & 10.69 & 2.21 & 0.15 & 0.08 & 0.07 & & 0.605 & 0.17 & 0.08 & 0.13 & 0.17 & 0.04 & 0.10 & 0.18 & 0.07 & 0.03 & 0.02 & 0.03 \\
\hline 11R4-17 & 242 & 10 & 48.01 & 1.12 & 15.73 & 10.86 & 0.19 & 9.40 & 10.99 & 2.16 & 0.13 & 0.07 & 0.06 & 2.26 & 0.607 & 0.27 & 0.08 & 0.22 & 0.31 & 0.02 & 0.11 & 0.16 & 0.09 & 0.02 & 0.02 & 0.01 \\
\hline 11R4-16 & 1233 & 10 & 48.42 & 1.16 & 16.19 & 11.48 & 0.19 & 9.06 & 11.18 & 2.19 & 0.12 & 0.07 & 0.08 & 2.29 & 0.584 & 0.11 & 0.05 & 0.15 & 0.20 & 0.03 & 0.10 & 0.15 & 0.07 & 0.02 & 0.01 & 0.03 \\
\hline 11R4-15 & 1223 & 8 & 48.75 & 1.26 & 16.20 & 10.78 & 0.19 & 8.44 & 11.28 & 2.18 & 0.22 & 0.15 & & 2.38 & 0.583 & 0.19 & 0.06 & 0.16 & 0.26 & 0.04 & 0.15 & 0.12 & 0.06 & 0.03 & 0.06 & 0.02 \\
\hline $11 \mathrm{R} 4-14$ & 1214 & $\begin{array}{l}8 \\
6\end{array}$ & 49.24 & $\begin{array}{l}1.20 \\
1.41\end{array}$ & $\begin{array}{l}15.20 \\
15.82\end{array}$ & $\begin{array}{l}10.78 \\
11.88\end{array}$ & $\begin{array}{l}0.19 \\
0.20\end{array}$ & $\begin{array}{l}8.44 \\
8.01\end{array}$ & 11.55 & 2.07 & 0.09 & 0.11 & & 2.37 & 0.5 & $\begin{array}{l}0.19 \\
0.19\end{array}$ & $\begin{array}{l}.00 \\
0.08\end{array}$ & & $\begin{array}{l}0.20 \\
0.29\end{array}$ & $\begin{array}{l}0.04 \\
0.03\end{array}$ & 14 & 0.19 & $\begin{array}{l}0.00 \\
0.10\end{array}$ & 0.02 & $\begin{array}{l}0.00 \\
0.03\end{array}$ & $\begin{array}{l}0.02 \\
0.05\end{array}$ \\
\hline $11 \mathrm{R} 4$ & 1205 & $\begin{array}{l}0 \\
8\end{array}$ & 49.39 & $\begin{array}{l}1.41 \\
1.50\end{array}$ & 15.77 & $\begin{array}{l}11.80 \\
12.10\end{array}$ & $\begin{array}{l}0.19 \\
0.19\end{array}$ & $\begin{array}{l}0.01 \\
7.50\end{array}$ & 11.49 & 2.52 & 0 & 0.17 & $\begin{array}{l}0.10 \\
0.07\end{array}$ & 2.37 & 0.5 & 0.15 & 0.07 & $\begin{array}{l}0.13 \\
0.12\end{array}$ & 0.57 & $\begin{array}{l}0.03 \\
0.03\end{array}$ & $\begin{array}{l}4 \\
8\end{array}$ & 0.15 & $\begin{array}{l}0.11 \\
0.11\end{array}$ & 0.04 & $\begin{array}{l}0.03 \\
0.04\end{array}$ & $\begin{array}{l}0.02 \\
0.02\end{array}$ \\
\hline 11 & 1197 & 7 & 49.08 & 1.54 & 14.74 & 13.19 & 0.21 & 7.02 & 11.78 & 2.3 & 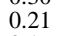 & 0.15 & 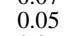 & 2.58 & 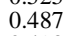 & 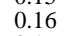 & 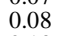 & & 0.39 & & & 015 & 0.12 & & & 4 \\
\hline 11 & 1186 & 7 & 49. & 1.85 & 14.21 & 12.64 & 0.2 & 7. & 1 & 2. & 0.16 & 0.1 & & 2. & 0.49 & 0.17 & 0.13 & 0. & 0.51 & 0.0 & & 0.12 & 9 & 3 & & 4 \\
\hline 11R4-6 & 1177 & 8 & 50.65 & 2.12 & 13.75 & 13.33 & 0.23 & 6.41 & 11.20 & 2.55 & 0.17 & 0.16 & 0.07 & 2.65 & 0.46 & 20 & 0.10 & 0.1 & 0.22 & 3 & & 0.18 & 8 & 2 & & 2 \\
\hline $11 \mathrm{R} 4-7$ & 1168 & 6 & 50.35 & 1.98 & 13.51 & 13.59 & 0.23 & $\begin{array}{l}0.41 \\
6.32\end{array}$ & 10.40 & 2.57 & 0.17 & 0.15 & 0.09 & 2.77 & 0.453 & 0.49 & 0.08 & 0.26 & 0.48 & $\begin{array}{l}0.05 \\
0.02\end{array}$ & 0.09 & $\begin{array}{l}0.10 \\
0.22\end{array}$ & $\begin{array}{l}0.05 \\
0.05\end{array}$ & 0.03 & 0.03 & 0.04 \\
\hline 11R4- & 58 & 7 & 50.22 & 2.70 & 12.77 & 14.00 & 0.24 & 5.65 & 10.20 & 2.49 & 0.27 & 0.20 & 0.05 & 2.79 & 0.418 & 0.20 & 0.07 & 0.16 & 0.15 & 0.02 & 0.08 & 0.17 & 0.09 & 0.02 & 0.03 & 0.03 \\
\hline $11 \mathrm{R} 4-10$ & 1148 & 7 & 51.01 & 3.43 & 12.20 & 15.04 & 0.26 & 5.06 & 9.85 & 2.50 & 0.24 & 0.23 & & 2.70 & 0.375 & 1.16 & 0.08 & 0.12 & 0.24 & 0.03 & 0.26 & 0.33 & 0.17 & 0.03 & 0.07 & \\
\hline 11R4-11 & 1136 & 6 & 50.21 & 3.83 & 11.92 & 15.14 & 0.31 & 5.27 & 9.31 & 2.30 & 0.29 & 0.30 & 0.07 & & 0.383 & 0.54 & 0.21 & 0.22 & 0.65 & 0.04 & 0.20 & 0.16 & 0.14 & 0.03 & 0.03 & 0.05 \\
\hline 11R4-12 & 1130 & 4 & 48.88 & 5.07 & 11.62 & $\begin{array}{l}15.24 \\
\text { - }\end{array}$ & 0.26 & 4.68 & 8.90 & 2.51 & 0.38 & 0.41 & 0.05 & & 0.354 & & 0.26 & 0.63 & 0.64 & 0.05 & 0.11 & 0.31 & 0.21 & 0.06 & 0.07 & 0.03 \\
\hline 11R4-13 & 1120 & 5 & 48.43 & 5.52 & 11.03 & 17.13 & 0.82 & 4.21 & 9.35 & 2.23 & 0.42 & 0.43 & 0.04 & & 0.305 & 0.37 & 0.23 & 0.49 & 0.61 & 0.05 & 0.27 & 0.05 & 0.09 & 0.05 & 0.05 & 0.02 \\
\hline \multicolumn{27}{|c|}{ 152-917A-86R-7, 45-49 cm (Piece 1) } \\
\hline $86 \mathrm{R} 7-16$ & 1233 & 5 & 49.56 & 1.03 & 16.81 & 9.84 & 0.15 & 8.73 & 11.04 & 2.58 & 0.36 & 0.15 & 0.06 & & 0.61 & 0.20 & 0.02 & 0.33 & 0.34 & 0.03 & 0.10 & 0.23 & 0.11 & 0.03 & 0.05 & 0.03 \\
\hline & & 10 & & & 16. & 9.35 & 0. & 8.4 & & 2. & 0. & 0.1 & 0.07 & 2.66 & 0.6 & & 0.0 & & 0.30 & 0.03 & & 0.18 & 0.09 & & 0.04 & 0.05 \\
\hline & & 7 & 50 & 1. & 15 & 10.55 & 0.2 & 7. & 11 & 2.6 & 0. & 0.1 & 0.03 & 2.8 & & & 0.07 & & 0.36 & 0.03 & 0. & 0.19 & 0.05 & 0.03 & & 0.02 \\
\hline & 1 & 6 & 50 & 1. & 15.10 & 11.52 & 0. & 7. & 11.34 & 2.5 & 0. & 0.1 & 0.06 & 2.7 & 0.5 & & 0.09 & 0. & 0.23 & 0.07 & 0.12 & 0.21 & 0.10 & 0.04 & 0.06 & 0.04 \\
\hline & 1186 & 6 & 50. & 1.4 & 14.56 & 11.52 & & 6.8 & 11.42 & 2.6 & & 0.1 & 0.06 & 2.7 & 0.5 & & 0.07 & 0.09 & 0.20 & 0.02 & 0.16 & 0.07 & 0.06 & 0.03 & 0.04 & 0.05 \\
\hline & & 7 & 50.8 & 1.57 & 14.23 & 11.82 & & & 11.23 & 2.6 & & 0.20 & & 2.8 & 0.4 & & & & 0.31 & & 0.05 & 0.18 & 0.12 & 0.04 & & 0.08 \\
\hline 8 & 68 & 5 & 50.86 & 1.75 & 14.08 & 12.19 & 0.22 & 5.96 & 10.43 & 2.9 & 0.57 & 0.18 & - & 3.19 & 0.4 & 0.10 & 0.03 & 0.09 & 0.18 & 0.02 & 0.10 & 0.13 & 0.07 & 0.03 & 0.05 & - \\
\hline $86 R-7-9$ & $\begin{array}{l}1158 \\
1158\end{array}$ & 8 & 50.88 & 2.36 & $\begin{array}{l}13.64 \\
\end{array}$ & 13.57 & 0.20 & 5.47 & 9.94 & 3.05 & 0.67 & 0.25 & 二 & & 0.418 & 0.17 & 0.08 & 0.09 & $\begin{array}{l}0.18 \\
0.18\end{array}$ & 0.02 & 0.10 & 0.10 & 0.09 & 0.04 & 4 & - \\
\hline $86 \mathrm{R} 7-10$ & $\begin{array}{l}1148 \\
1148\end{array}$ & $\begin{array}{l}8 \\
6\end{array}$ & 51.85 & $\begin{array}{l}2.50 \\
2.72\end{array}$ & 13.44 & 14.16 & 0.21 & $\begin{array}{l}5.41 \\
5.07\end{array}$ & 9.56 & 3.04 & 0.81 & 0.3 & - & & $\begin{array}{l}0.410 \\
0.390\end{array}$ & & $\begin{array}{l}0.08 \\
0.08\end{array}$ & 0.11 & $\begin{array}{l}0.10 \\
0.45\end{array}$ & $\begin{array}{l}0.02 \\
0.04\end{array}$ & & 0.15 & $\begin{array}{l}0.09 \\
0.06\end{array}$ & $\begin{array}{l}0.04 \\
0.07\end{array}$ & & 二 \\
\hline 27-11 & 113 & 6 & 52. & 2.67 & 12.98 & 14.13 & 0.25 & 5. & 9.34 & 2.6 & 0.74 & 0.3 & - & & 0.3 & & 0. & 0. & 0.54 & 0. & 0 . & 0.24 & 0.25 & 8 & & - \\
\hline 2 & 113 & 9 & 52. & 3.0 & 12. & 13 & 0. & 4. & & 2. & 0. & 0.4 & - & & 0.3 & & 0. & & & 0. & & & 0.20 & & & - \\
\hline & 1 & 6 & & $J$ & & & 0. & & & 2.8 & & 0. & - & & & & & & & & & & 0. & & & - \\
\hline 86R7-8 & 1111 & 5 & 51.52 & 3.83 & 12.62 & $\begin{array}{l}14.42 \\
\text {. }\end{array}$ & 0.23 & $\begin{array}{l}4.156 \\
3.76\end{array}$ & $\begin{array}{l}0.10 \\
7.72\end{array}$ & 2.38 & $\begin{array}{l}1.34 \\
1.34\end{array}$ & 0.63 & 二 & 2.48 & 0.317 & 0.56 & 0.12 & 0.15 & 0.26 & 0.03 & 0.09 & 0.14 & 0.16 & 0.08 & 0.09 & 二 \\
\hline
\end{tabular}

Notes: Averages of individual analyses and associated 1 standard deviations. $\mathrm{N}=$ number of analyses used for calculating each average. $\mathrm{FeO} *=$ total iron as $\mathrm{FeO} . \quad-=$ below detection limit. $\mathrm{Mg} \#=\mathrm{Mg} /(\mathrm{Mg}+\mathrm{Fe})$ calculated with all iron as $\mathrm{FeO}$. Corrected $\mathrm{Na}_{2} \mathrm{O}$ values are based on the estimated losses from Table 2. 
Table 4. Composition of experimental olivine.

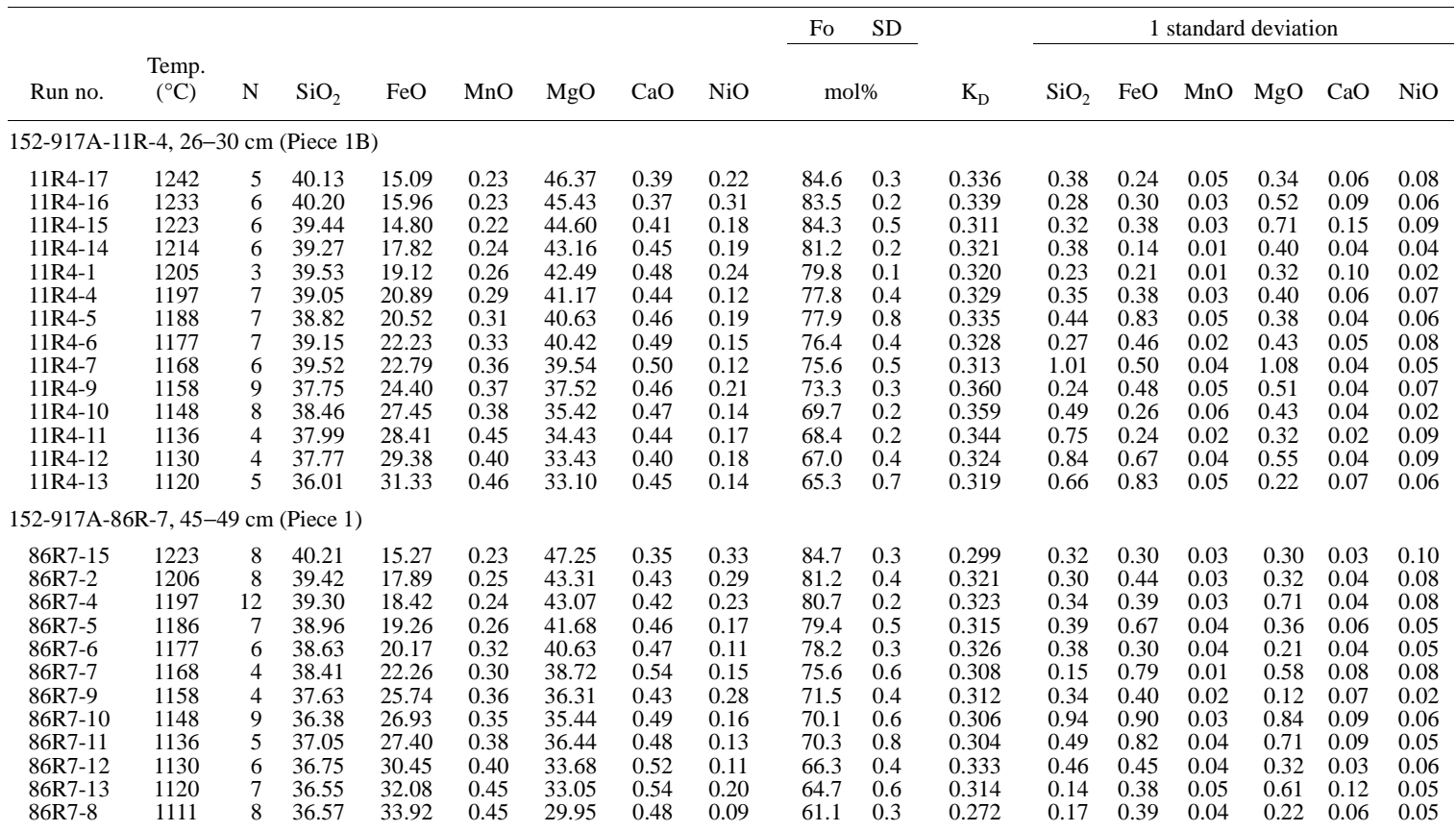

Notes: Averages of individual analyses and associated 1 standard deviations. $\mathrm{N}=$ number of analyses. $\mathrm{SD}=1$ standard deviation of Fo. $\mathrm{K}_{\mathrm{D}}$ is calculated as discussed in text.

Table 5. Composition of experimental plagioclase.

\begin{tabular}{|c|c|c|c|c|c|c|c|c|c|c|c|c|c|c|c|c|c|c|c|c|}
\hline \multirow[b]{2}{*}{$\begin{array}{c}\text { Run } \\
\text { no. }\end{array}$} & \multirow[b]{2}{*}{$\begin{array}{c}\text { Temp. } \\
\left({ }^{\circ} \mathrm{C}\right)\end{array}$} & \multirow[b]{2}{*}{$\mathrm{N}$} & \multirow[b]{2}{*}{$\mathrm{SiO}_{2}$} & \multirow[b]{2}{*}{$\mathrm{TiO}_{2}$} & \multirow[b]{2}{*}{$\mathrm{Al}_{2} \mathrm{O}_{3}$} & \multirow[b]{2}{*}{$\mathrm{FeO}$} & \multirow[b]{2}{*}{$\mathrm{MgO}$} & \multirow[b]{2}{*}{$\mathrm{CaO}$} & \multirow[b]{2}{*}{$\mathrm{Na}_{2} \mathrm{O}$} & \multirow[b]{2}{*}{$\mathrm{K}_{2} \mathrm{O}$} & \multirow{2}{*}{\multicolumn{2}{|c|}{$\begin{array}{l}\text { An SD } \\
\text { mol\% }\end{array}$}} & \multicolumn{8}{|c|}{1 standard deviation } \\
\hline & & & & & & & & & & & & & $\mathrm{SiO}_{2}$ & $\mathrm{TiO}_{2}$ & $\mathrm{Al}_{2} \mathrm{O}_{3}$ & $\mathrm{FeO}$ & $\mathrm{MgO}$ & $\mathrm{CaO}$ & $\mathrm{Na}_{2} \mathrm{O}$ & $\mathrm{K}_{2} \mathrm{O}$ \\
\hline \multicolumn{21}{|c|}{ 152-917A-11R-4, 26-30 cm (Piece 1B) } \\
\hline 11R4-14 & 1214 & 8 & 49.26 & 0.06 & 32.03 & 0.88 & 0.24 & 15.57 & 2.46 & 0.02 & 77.7 & 1.5 & 0.40 & 0.03 & 0.29 & 0.10 & 0.03 & 0.25 & 0.19 & 0.01 \\
\hline 11R4-1 & 1205 & 6 & 50.52 & 0.08 & 32.49 & 1.01 & 0.31 & 15.19 & 2.69 & 0.05 & 75.5 & 3.2 & 0.84 & 0.03 & 0.58 & 0.10 & 0.04 & 0.74 & 0.33 & 0.02 \\
\hline $11 \mathrm{R} 4-4$ & 1197 & 6 & 49.94 & 0.05 & 32.84 & 0.97 & 0.24 & 15.45 & 2.68 & 0.04 & 75.9 & 1.3 & 0.31 & 0.02 & 0.27 & 0.16 & 0.03 & 0.39 & 0.15 & 0.02 \\
\hline 11R4-5 & 1186 & 6 & 49.26 & 0.09 & 31.07 & 1.08 & 0.28 & 14.53 & 2.76 & 0.04 & 74.2 & 3.1 & 0.84 & 0.04 & 0.41 & 0.18 & 0.05 & 0.69 & 0.31 & 0.01 \\
\hline 11R4-6 & 1177 & 6 & 51.79 & 0.07 & 31.47 & 1.16 & 0.24 & 14.36 & 3.18 & 0.03 & 71.3 & 5.4 & 0.03 & 1.27 & 0.07 & 0.03 & 1.26 & 0.57 & 0.01 & \\
\hline $11 \mathrm{R} 4-7$ & 1168 & 10 & 50.60 & 0.07 & 31.82 & 1.11 & 0.24 & 14.41 & 2.94 & 0.03 & 72.9 & 4.4 & 1.18 & 0.03 & 0.77 & 0.14 & 0.03 & 0.93 & 0.47 & 0.01 \\
\hline 11R4-9 & 1158 & 9 & 50.67 & 0.06 & 31.44 & 1.18 & 0.26 & 14.40 & 3.06 & 0.06 & 72.0 & 4.0 & 0.99 & 0.03 & 0.61 & 0.13 & 0.05 & 0.80 & 0.44 & 0.02 \\
\hline 11R4-10 & 1148 & 6 & 52.72 & 0.11 & 30.70 & 1.20 & 0.27 & 13.56 & 3.50 & 0.05 & 68.0 & 2.1 & 0.64 & 0.03 & 0.37 & 0.10 & 0.03 & 0.32 & 0.26 & 0.01 \\
\hline 11R4-11 & 1136 & 2 & 53.53 & 0.11 & 28.48 & 1.22 & 0.24 & 12.53 & 4.19 & 0.05 & 62.1 & & & & & & & & & \\
\hline $4-12$ & 1130 & 3 & 53.26 & 0.12 & 28.79 & 1.20 & 0.26 & 12.38 & 4.07 & 0.08 & 62.4 & 7.7 & 2.27 & 0.03 & 1.24 & & & 1.30 & 0.89 & 0.05 \\
\hline 11R4-13 & 1120 & 7 & 53.65 & 0.11 & 28.77 & 1.11 & 0.26 & 11.65 & 4.26 & 0.10 & 59.8 & 6.3 & 1.64 & 0.05 & 1.29 & 0.17 & 0.10 & 1.36 & 0.61 & 0.04 \\
\hline \multicolumn{21}{|c|}{ 152-917A-86R-7, 45-49 cm (Piece 1) } \\
\hline $86 R 7-15$ & 1223 & 10 & 49.02 & 0.07 & 33.37 & 0.69 & 0.26 & 16.25 & 2.32 & 0.06 & 79.2 & 0.9 & 0.32 & 0.02 & 0.16 & 0.12 & 0.02 & 0.28 & 0.10 & 0.02 \\
\hline & 1206 & 10 & 49.75 & 0.06 & 32.28 & 0.80 & 0.31 & 15.16 & 2.56 & 0.09 & 76.2 & 2.7 & 0.61 & 0.04 & 0.56 & 0.10 & 0.09 & 0.64 & 0.29 & 0.02 \\
\hline $86 R 7-4$ & 1197 & 8 & 51.02 & 0.08 & 31.67 & 0.98 & 0.19 & 14.17 & 3.03 & 0.11 & 71.6 & 2.2 & 0.53 & 0.03 & 0.52 & 0.15 & 0.05 & 0.51 & 0.22 & 0.04 \\
\hline $86 \mathrm{R} 7-5$ & 1186 & 4 & 51.05 & 0.11 & 31.29 & 1.01 & 0.28 & 13.71 & 3.07 & 0.12 & 70.6 & 2.3 & 0.82 & 0.03 & 0.20 & 0.12 & 0.12 & 0.33 & 0.27 & 0.02 \\
\hline $86 \mathrm{R} 7-6$ & 1177 & 4 & 50.77 & 0.06 & 31.28 & 0.99 & 0.21 & 13.89 & 3.28 & 0.10 & 69.6 & 4.3 & 1.04 & 0.03 & 0.55 & 0.15 & 0.05 & 0.88 & 0.46 & 0.03 \\
\hline $86 \mathrm{R} 7-7$ & 1168 & 4 & 50.30 & 0.09 & 31.62 & 1.08 & 0.14 & 13.73 & 3.13 & 0.12 & 70.3 & 4.1 & 1.16 & 0.04 & 0.97 & 0.10 & 0.10 & 0.88 & 0.43 & 0.01 \\
\hline $86 \mathrm{R} 7-9$ & 1158 & 4 & 51.62 & 0.09 & 30.43 & 1.07 & 0.22 & 13.33 & 3.85 & 0.15 & 65.1 & 5.0 & 1.34 & 0.03 & 0.91 & 0.23 & 0.05 & 1.07 & 0.56 & 0.02 \\
\hline $86 R 7-10$ & 1148 & 9 & 53.11 & 0.07 & 29.16 & 0.96 & 0.20 & 12.13 & 4.27 & 0.17 & 60.5 & 6.1 & 1.62 & 0.04 & 1.34 & 0.09 & 0.04 & 1.29 & 0.63 & 0.06 \\
\hline $7-11$ & 1136 & 5 & 53.99 & 0.18 & 28.54 & 1.01 & 0.25 & 11.63 & 4.42 & 0.19 & 58.6 & 2.9 & 0.54 & 0.08 & 0.26 & 0.27 & 0.13 & 0.60 & 0.31 & 0.03 \\
\hline $7-12$ & 1130 & 10 & 53.17 & 0.10 & 29.29 & 0.99 & 0.16 & 12.22 & 4.28 & 0.20 & 60.5 & 4.1 & 1.20 & 0.06 & 0.65 & 0.14 & 0.07 & 0.77 & 0.46 & 0.05 \\
\hline $86 R 7-13$ & 1120 & 13 & 53.12 & 0.10 & 29.35 & 0.97 & 0.16 & 12.19 & 4.21 & 0.20 & 60.8 & 6.8 & 1.88 & 0.06 & 1.29 & 0.12 & 0.03 & 1.41 & 0.70 & 0.05 \\
\hline 86R7-8 & 1111 & 4 & 53.27 & 0.09 & 30.08 & 1.09 & 0.12 & 12.11 & 4.34 & 0.27 & 59.7 & 6.2 & 1.58 & 0.03 & 1.25 & 0.07 & 0.02 & 1.30 & 0.65 & 0.05 \\
\hline
\end{tabular}

Notes: Averages of individual analyses and associated 1 standard deviations. $\mathrm{N}=$ number of analyses. SD is 1 standard deviation of An.

(Papike et al., 1974), the $\mathrm{Fe}^{3+}$ content of the $11 \mathrm{R} 4$ augites is $0.034 \pm$ 0.010 per formula unit, while the $\mathrm{Fe}^{3+}$ content of $86 \mathrm{R} 7$ augites is $0.046 \pm 0.013$ per formula unit. Using these values, the $K_{\mathrm{D}}^{\mathrm{Fe}^{2+} / \mathrm{Mg}}[\mathrm{cpx} / \mathrm{liq}]$ is $0.238 \pm 0.025$ (Fig. 3).

\section{Liquid Lines of Descent}

The experimental results presented in Table 3 can be used to define the low pressure liquid lines of descent (LLD) for 11R4 and 86R7. The composition of the liquids as a function of $\mathrm{MgO}$ content is shown in Figure 4. The LLD for the two samples are broadly sim- ilar, although there are several important differences. For example, the $\mathrm{SiO}_{2}$ content gradually increases with decreasing $\mathrm{MgO}$ to 5-6 $\mathrm{wt} \%$. However at more evolved stages, the LLDs diverge with the 86R7 liquids evolving toward higher silica content and the 11R4 liquids evolving to lower silica content. For $11 \mathrm{R} 4, \mathrm{Al}_{2} \mathrm{O}_{3}$ first increases with decreasing $\mathrm{MgO}$, which reflects olivine crystallization prior to plagioclase appearance, and subsequently falls with co-crystallization of plagioclase, olivine, and augite, coinciding with the LLD for 86R7 (Fig. 4). In contrast, $\mathrm{CaO}$ content of experimental liquids gradually increases with decreasing $\mathrm{MgO}$ during co-crystallization of olivine and plagioclase but decreases below $\sim 7 \mathrm{wt} \% \mathrm{MgO}$ 
Table 6. Composition of experimental pyroxene.

\begin{tabular}{|c|c|c|c|c|c|c|c|c|c|c|c|c|c|c|c|c|c|c|c|c|c|c|c|c|c|c|}
\hline \multirow[b]{2}{*}{ Run no. } & \multirow{2}{*}{$\begin{array}{l}\text { Temp. } \\
\left({ }^{\circ} \mathrm{C}\right)\end{array}$} & \multirow[b]{2}{*}{$\mathrm{N}$} & \multirow[b]{2}{*}{$\mathrm{SiO}_{2}$} & \multirow[b]{2}{*}{$\mathrm{TiO}_{2}$} & \multirow[b]{2}{*}{$\mathrm{Al}_{2} \mathrm{O}_{3}$} & \multirow[b]{2}{*}{$\mathrm{FeO}$} & \multirow[b]{2}{*}{$\mathrm{MnO}$} & \multirow[b]{2}{*}{$\mathrm{MgO}$} & \multirow[b]{2}{*}{$\mathrm{CaO}$} & \multirow[b]{2}{*}{$\mathrm{Na}_{2} \mathrm{O}$} & \multirow[b]{2}{*}{$\mathrm{Cr}_{2} \mathrm{O}_{3}$} & \multirow{2}{*}{ En } & \multirow{2}{*}{$\frac{\mathrm{Fs}}{\mathrm{mol} \%}$} & \multirow[t]{2}{*}{ Wo } & \multirow[b]{2}{*}{ Mg\# } & \multirow[b]{2}{*}{ SD } & \multirow[b]{2}{*}{$\mathrm{K}_{\mathrm{D}}$} & \multicolumn{9}{|c|}{1 standard deviation } \\
\hline & & & & & & & & & & & & & & & & & & $\mathrm{SiO}_{2}$ & $\mathrm{TiO}_{2}$ & $\mathrm{Al}_{2} \mathrm{O}_{3}$ & $\mathrm{FeO}$ & $\mathrm{MnO}$ & $\mathrm{MgO}$ & $\mathrm{CaO}$ & $\mathrm{Na}_{2} \mathrm{O}$ & $\mathrm{Cr}_{2} \mathrm{O}_{3}$ \\
\hline \multicolumn{27}{|c|}{ 152-917A-11R-4, 26-30 cm (Piece 1B) } \\
\hline $11 \mathrm{R} 4-6$ & 1177 & 7 & 51.28 & 0.75 & 3.66 & 8.05 & 0.18 & 15.66 & 20.35 & 0.25 & 0.51 & 45.0 & 13.0 & 42.0 & 77.6 & 1.7 & 0.254 & 0.96 & 0.16 & 0.91 & 0.50 & 0.03 & 0.86 & 1.00 & 0.04 & 0.18 \\
\hline $\begin{array}{l}11 \mathrm{R} 4-7 \\
11 \mathrm{R}-9\end{array}$ & $\begin{array}{l}1168 \\
1158\end{array}$ & $\begin{array}{r}5 \\
13\end{array}$ & $\begin{array}{l}51.21 \\
50.52\end{array}$ & $\begin{array}{l}0.84 \\
0.84\end{array}$ & $\begin{array}{l}3.80 \\
3.59\end{array}$ & $\begin{array}{l}8.39 \\
8.92\end{array}$ & $\begin{array}{l}0.19 \\
0.21\end{array}$ & $\begin{array}{l}15.68 \\
15.56\end{array}$ & $\begin{array}{l}19.56 \\
19.46\end{array}$ & $\begin{array}{l}0.27 \\
0.27\end{array}$ & $\begin{array}{l}0.34 \\
0.30\end{array}$ & $\begin{array}{l}45.5 \\
45.0\end{array}$ & $\begin{array}{l}13.7 \\
14.5\end{array}$ & $\begin{array}{l}40.8 \\
40.5\end{array}$ & $\begin{array}{l}76.9 \\
75.7\end{array}$ & $\begin{array}{l}1.7 \\
2.2\end{array}$ & $\begin{array}{l}0.270 \\
0.232\end{array}$ & $\begin{array}{l}1.02 \\
0.93\end{array}$ & $\begin{array}{l}0.25 \\
0.15\end{array}$ & $\begin{array}{l}1.09 \\
0.73\end{array}$ & $\begin{array}{l}0.64 \\
0.89\end{array}$ & $\begin{array}{l}0.03 \\
0.03\end{array}$ & $\begin{array}{l}1.03 \\
0.91\end{array}$ & $\begin{array}{l}0.76 \\
0.81\end{array}$ & $\begin{array}{l}0.07 \\
0.06\end{array}$ & $\begin{array}{l}0.24 \\
0.13\end{array}$ \\
\hline $11 R 4-10$ & 1148 & $\begin{array}{l}13 \\
16\end{array}$ & 51.31 & $\begin{array}{l}0.84 \\
0.92\end{array}$ & $\begin{array}{l}3.59 \\
3.75\end{array}$ & $\begin{array}{l}8.92 \\
9.42\end{array}$ & $\begin{array}{l}0.21 \\
0.27\end{array}$ & $\begin{array}{l}15.50 \\
15.10\end{array}$ & $\begin{array}{l}19.40 \\
19.36\end{array}$ & $\begin{array}{l}0.27 \\
0.27\end{array}$ & 0.25 & 44.0 & $\begin{array}{l}14.3 \\
15.4\end{array}$ & $\begin{array}{l}40.5 \\
40.6\end{array}$ & 74.1 & $\begin{array}{l}2.2 \\
2.7\end{array}$ & 0.252 & $\begin{array}{l}0.93 \\
0.73\end{array}$ & $\begin{array}{l}0.15 \\
0.16\end{array}$ & $\begin{array}{l}0.13 \\
0.62\end{array}$ & 0.89 & $\begin{array}{l}0.03 \\
0.04\end{array}$ & 0.91 & $\begin{array}{l}0.81 \\
070\end{array}$ & 0.06 & 0.13 \\
\hline 11R4-11 & $\begin{array}{l}1136 \\
1136\end{array}$ & $\begin{array}{r}10 \\
7\end{array}$ & 50.41 & 0.90 & 3.86 & 9.59 & 0.20 & 14.80 & 19.76 & 0.31 & 0.23 & 43.0 & 15.7 & 41.3 & 73.3 & 2.8 & 0.228 & 0.55 & 0.17 & 0.56 & $\begin{array}{l}1.11 \\
1.29\end{array}$ & $\begin{array}{l}0.04 \\
0.07\end{array}$ & $\begin{array}{l}0.01 \\
0.30\end{array}$ & 0.35 & $\begin{array}{l}0.04 \\
0.04\end{array}$ & $\begin{array}{l}0.11 \\
0.07\end{array}$ \\
\hline 11R4-12 & 1130 & 14 & 50.33 & 0.93 & 3.36 & 9.83 & 0.20 & 15.16 & 19.39 & 0.27 & 0.19 & 43.8 & 15.9 & 40.3 & 73.3 & 4.3 & 0.199 & 1.09 & 0.25 & 0.96 & 1.87 & 0.03 & 0.90 & $\begin{array}{l}0.02 \\
\end{array}$ & 0.05 & 0.08 \\
\hline $11 \mathrm{R} 4-13$ & 1120 & 5 & 49.73 & 1.35 & 2.25 & 13.81 & 0.36 & 14.78 & 16.20 & 0.28 & 0.18 & 43.3 & 22.7 & 34.1 & 65.6 & 7.7 & 0.245 & 0.95 & 0.29 & 0.69 & 2.61 & 0.08 & 1.69 & 0.73 & 0.03 & 0.09 \\
\hline \multicolumn{27}{|c|}{ 152-917A-86R-7, 45-49 cm (Piece 1) } \\
\hline 86R7-6 & 1177 & 8 & 52.23 & 0.53 & 2.18 & 7.01 & 0.18 & 16.56 & 20.31 & 0.28 & 0.56 & 47.2 & 11.2 & 41.6 & 80.8 & 2.8 & 0.244 & 0.53 & 0.11 & 0.50 & 1.20 & 0.05 & 0.36 & 0.66 & 0.03 & 0.19 \\
\hline 86R7-7 & 1168 & 6 & 52.18 & 0.61 & 2.47 & 7.62 & 0.20 & 16.29 & 18.97 & 0.28 & 0.15 & 47.6 & 12.5 & 39.9 & 79.2 & 1.8 & & 0.61 & 0.13 & 0.52 & 0.61 & 0.02 & 0.42 & 1.05 & 0.07 & 0.10 \\
\hline $86 \mathrm{R} 7-9$ & 1158 & 7 & 51.38 & 0.56 & 2.34 & 7.64 & 0.16 & 16.04 & 20.23 & 0.29 & 0.38 & 46.0 & 12.3 & 41.7 & 78.9 & 1.6 & 0.192 & 0.78 & 0.10 & 0.41 & 0.59 & 0.03 & 0.56 & 0.44 & 0.07 & 0.14 \\
\hline $86 \mathrm{R} 7-10$ & 1148 & 11 & 51.38 & 0.72 & 2.21 & 8.88 & 0.21 & 16.50 & 19.56 & 0.30 & 0.18 & 46.4 & 14.0 & 39.6 & 76.8 & 3.2 & 0.177 & 1.27 & 0.17 & 0.36 & 1.35 & 0.05 & 0.56 & 0.73 & 0.05 & 0.11 \\
\hline 86R7-11 & 1136 & 8 & 51.58 & 0.72 & 2.30 & 8.90 & 0.22 & 16.26 & 19.48 & 0.29 & 0.14 & 46.1 & 14.2 & 39.7 & 76.5 & 3.2 & 0.192 & 0.63 & 0.18 & 0.62 & 1.38 & 0.04 & 0.63 & 0.69 & 0.01 & 0.14 \\
\hline $86 \mathrm{R} 7-12$ & 1130 & 5 & 51.1 & 0.86 & 2.01 & 9.63 & 0.23 & 16.09 & 19.26 & 0.27 & 0.06 & 45.5 & 15.3 & 39.2 & 74.9 & 2.8 & 0.197 & 0.47 & 0.06 & 0.49 & 1.46 & 0.05 & 0.12 & 0.89 & 0.04 & 0.02 \\
\hline $86 \mathrm{R} 7-13$ & 1120 & 4 & 50. & 1.11 & 2.14 & 11.60 & 0.27 & 15.84 & 18.23 & 0.33 & 0.04 & 44.7 & 18.4 & 37.0 & 70.9 & 2.5 & 0.230 & 0.40 & 0.21 & 0.10 & 1.56 & 0.07 & 0.25 & 1.65 & 0.05 & 0.02 \\
\hline $86 \mathrm{R} 7-8$ & 1111 & 6 & 51.07 & 0.94 & 2.03 & 11.65 & 0.27 & 15.17 & 18.31 & 0.39 & - & 43.5 & 18.8 & 37.8 & 69.9 & 3.0 & 0.202 & 0.32 & 0.21 & 0.39 & 1.67 & 0.06 & 0.28 & 1.59 & 0.04 & - \\
\hline
\end{tabular}

Notes: Averages of individual analyses and associated 1 standard deviations. $\mathrm{N}=$ number of analyses. $\mathrm{Mg} \#=\mathrm{Mg} /(\mathrm{Mg}+\mathrm{Fe})$ calculated on atomic basis. $\mathrm{SD}=1 \mathrm{standard}$ deviation of $\mathrm{Mg} \#$. En $=$ enstatite, $\mathrm{Fs}=$ ferrosilite, $\mathrm{Wo}=$ wollastonite, all on a molecular basis. $\mathrm{K}_{\mathrm{D}}$ is calculated as discussed in text. $-=$ below detection limit. 

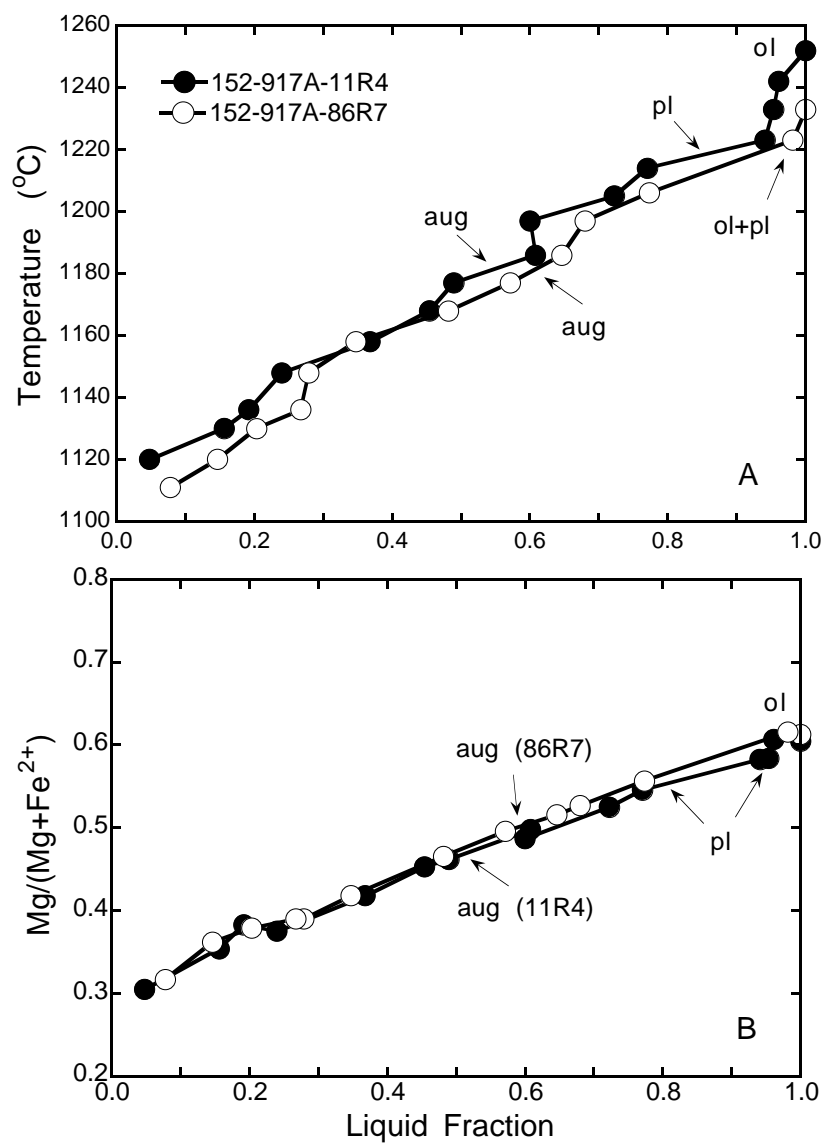

Figure 1. Melting temperature and liquid $\mathrm{Mg} /\left(\mathrm{Mg}+\mathrm{FeO}_{\text {total }}\right)$ ratio as a function of liquid fraction (Table 2). Abbreviations: $\mathrm{pl}=$ plagioclase, $\mathrm{ol}=$ olivine, and aug = augite. A. Temperature vs. liquid fraction. B. $\mathrm{Mg} /(\mathrm{Mg}+\mathrm{Fe})$ ratio vs. liquid fraction.

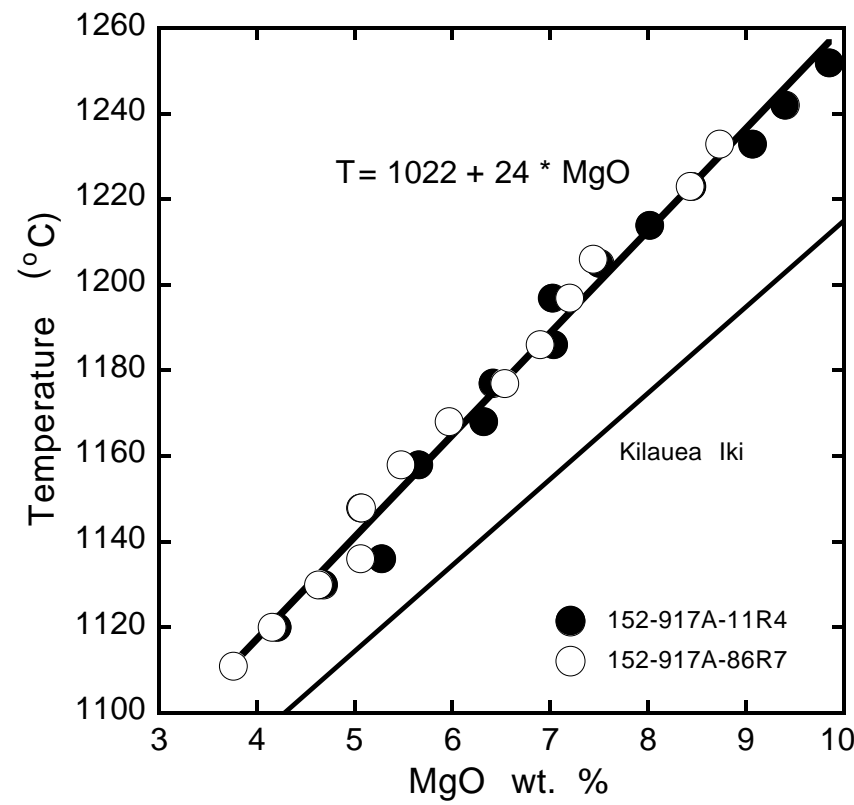

Figure 2. Melting temperature $\left({ }^{\circ} \mathrm{C}\right)$ as a function of liquid $\mathrm{MgO}(\mathrm{wt} \%)$ concentrations. The best fit to the data is described by $\mathrm{T}=1022+24 \cdot \mathrm{MgO}$. For comparison is shown the regression obtained by Helz and Thornber (1987) on Kilauea Iki lava lake samples $(\mathrm{T}=1014+20.1 \cdot \mathrm{MgO})$.

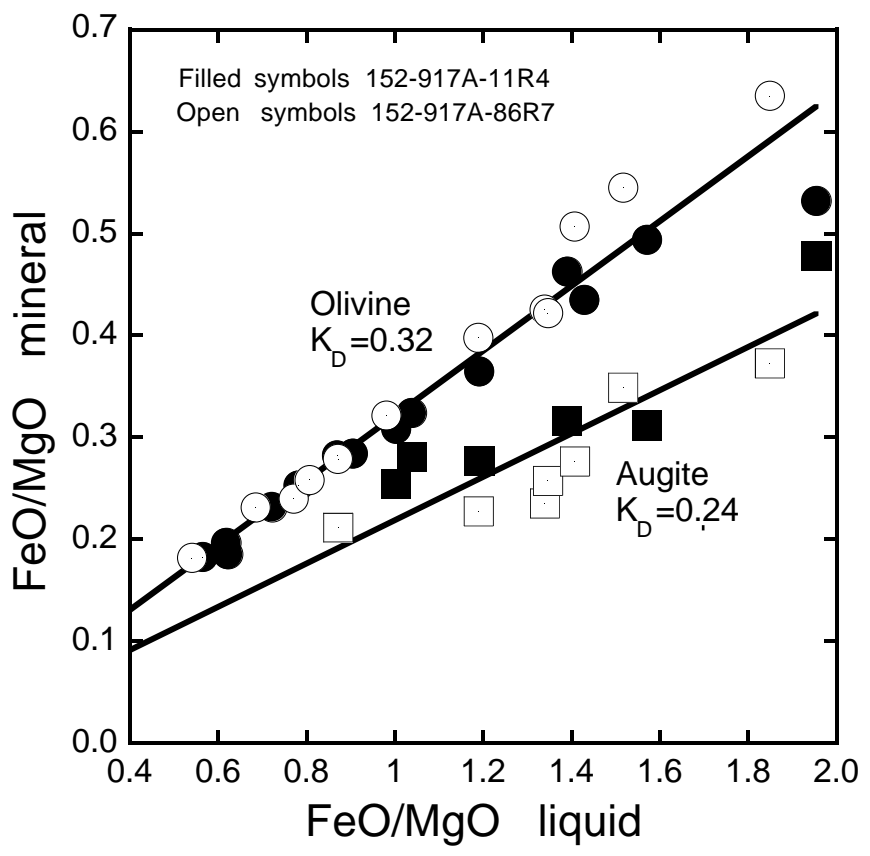

Figure 3. $\mathrm{FeO} / \mathrm{MgO}$ for olivine and augite vs. $\mathrm{FeO} / \mathrm{MgO}$ for coexisting liquid (mole proportions). The ratio of $\mathrm{Fe}^{3+} / \mathrm{Fe}^{2+}$ of the liquid is calculated using relations presented by Kilinc et al. (1983). The $K_{\mathrm{D}}$ for olivine is $0.322 \pm$ 0.018 and for augite is $0.238 \pm 0.025$ ( \pm 1 S.D.).

with crystallization of augite. As expected, $\mathrm{FeO}$ remains fairly constant with only olivine crystallization, but, as seen for the 11R4 and 86R7 LLDs, steadily increases during co-crystallization of olivine and plagioclase, as well as with the appearance of augite. The $\mathrm{Na}_{2} \mathrm{O}$ $\mathrm{MgO}$ relations are less systematic, but $\mathrm{Na}_{2} \mathrm{O}$ generally increases with crystallization of olivine and plagioclase and is depleted in more evolved liquids. The oxides $\mathrm{TiO}_{2}, \mathrm{P}_{2} \mathrm{O}_{5}$, and $\mathrm{K}_{2} \mathrm{O}$ show exponentially increasing concentrations with decreasing $\mathrm{MgO}$, indicating that these oxides behave incompatibly over the entire crystallization interval.

An important difference between the LLD of the two bulk compositions can be seen in the variation of the $\mathrm{CaO} / \mathrm{Al}_{2} \mathrm{O}_{3}$ ratio with $\mathrm{MgO}$ content (Fig. 5). Fractionation of olivine has little effect on $\mathrm{CaO} / \mathrm{Al}_{2} \mathrm{O}_{3}$, while the ratio is sensitive to the proportions of plagioclase and clinopyroxene crystallizing. It can be shown, based on the data presented in Table 2, that the equilibrium modes for $86 \mathrm{R} 7$ are slightly more plagioclase rich than for $11 \mathrm{R} 4$. The effects of plagioclase crystallization $\left(\mathrm{CaO} / \mathrm{Al}_{2} \mathrm{O}_{3}\right.$ of $\left.\sim 0.45\right)$ can be seen by the increase in $\mathrm{CaO} / \mathrm{Al}_{2} \mathrm{O}_{3}$ ratio beginning at $\sim 9 \mathrm{wt} \% \mathrm{MgO}$. When augite appears at $6-7 \mathrm{wt} \% \mathrm{MgO}$ the slopes change. The $\mathrm{CaO} / \mathrm{Al}_{2} \mathrm{O}_{3}$ ratio of augite produced in experiments on $86 \mathrm{R} 7$ ranges from 7.7 to 9.6 (Table 6), which is significantly greater than the value for cotectic liquids. As such, crystallization of plagioclase and augite leads to a decrease in the $\mathrm{CaO} / \mathrm{Al}_{2} \mathrm{O}_{3}$ ratio with decreasing $\mathrm{MgO}$. In contrast, $11 \mathrm{R} 4$ cotectic liquids evolve toward a modest increase in $\mathrm{CaO} / \mathrm{Al}_{2} \mathrm{O}_{3}$, reflecting crystallization of augite with a lower $\mathrm{CaO} / \mathrm{Al}_{2} \mathrm{O}_{3}$ ratio (5.1-5.8; Table $6)$. The small differences in the plagioclase mode for the two samples will counteract the differences in augite composition. Therefore, it is significant that only minor differences in augite composition will lead to detectable deviations in the LLD for multisaturated liquids.

Figures $6 \mathrm{~A}$ and $\mathrm{B}$ compare the experimental results with calculated positions of low pressure liquidus boundaries involving olivine, plagioclase, and augite as ternary projections from the normative plagioclase and olivine components (Longhi, 1991). The sensitivity of liquidus fields to changes in $\mathrm{Mg} \#$ of the liquid can be appreciated by comparing the projected cotectics for $\mathrm{Mg \# s}$ within the range of the experimental liquids $(0.61-0.32)$. In both projections, the olivineplagioclase-augite cotectic shifts slightly away from augite with de- 
creasing Mg\#. Similarly, the olivine-low-Ca pyroxene-plagioclase boundary shifts away from olivine and plagioclase with decreasing

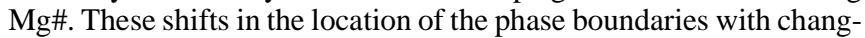
es in compositional parameters such as $\mathrm{Mg \#}$ are important to bear in mind when considering the phase relations for any evolving magmatic system. The parameterization presented by Longhi (1991) provides a graphical method for evaluating the magnitude of compositional effects on liquidus boundaries. The utility of the parameterization for evaluating liquidus relations for Leg 152 lavas is supported by the excellent agreement between the predicted location of the liquidus boundaries and the composition of multisaturated experimental liquids determined in this study.

\section{DISCUSSION}

\section{Experimental Liquid Lines of Descent and Leg 152 Lavas}

The importance of low pressure crystal fractionation for the evolution of Leg 152 magmas can be evaluated by comparing the exper- imental liquid lines of descent with the lava compositions given by Larsen et al. (Chap. 27, this volume). For example, the covariations among the major oxides are shown in Figure 4. In addition to the Lower, Middle, and Upper Series lavas from Site 917, Figure 4 shows the composition of the igneous units from Sites 915 and 918. These latter units are stratigraphically above the continental succession drilled at Site 917 and are considered part of the oceanic succession (Larsen, Saunders, Clift, et al., 1994). They are included here for completeness and to contrast the observed differentiation trends for the continental and oceanic successions.

Before proceeding, it is important to emphasize that the experimentally determined LLDs only provide constraints on equilibrium (closed system) crystallization. These paths will be similar to the paths for fractional crystallization during the early stages of magma evolution, but diverge for highly fractionated liquids. Furthermore, the results are limited to low pressure conditions and, therefore, divergent trends may be related to other factors including the pressure of crystallization, mixing, contamination, and primary melt composition. We consider these latter issues after first appraising the impor-
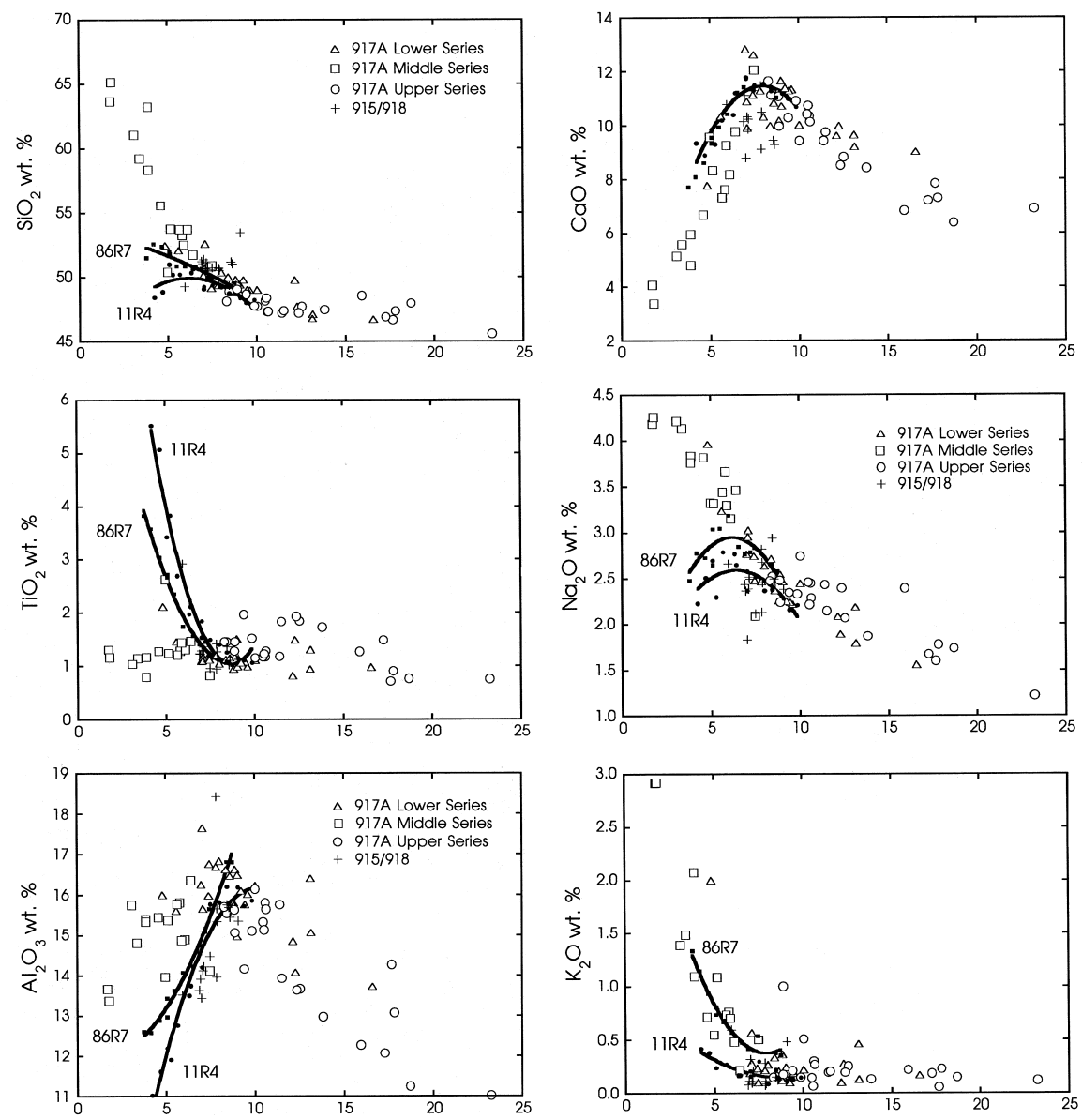

Figure 4. Compositions of the experimental liquids (Table 2) and the Leg 152 whole rock samples (Larsen et al., Chap. 27, this volume) plotted against $\mathrm{MgO}$. All analyses are recalculated on an anhydrous basis with all iron as $\mathrm{FeO}$.
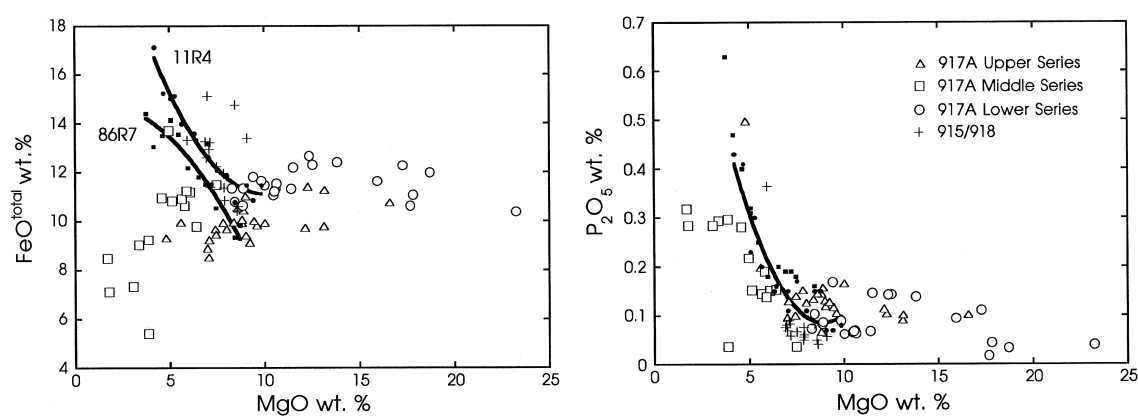


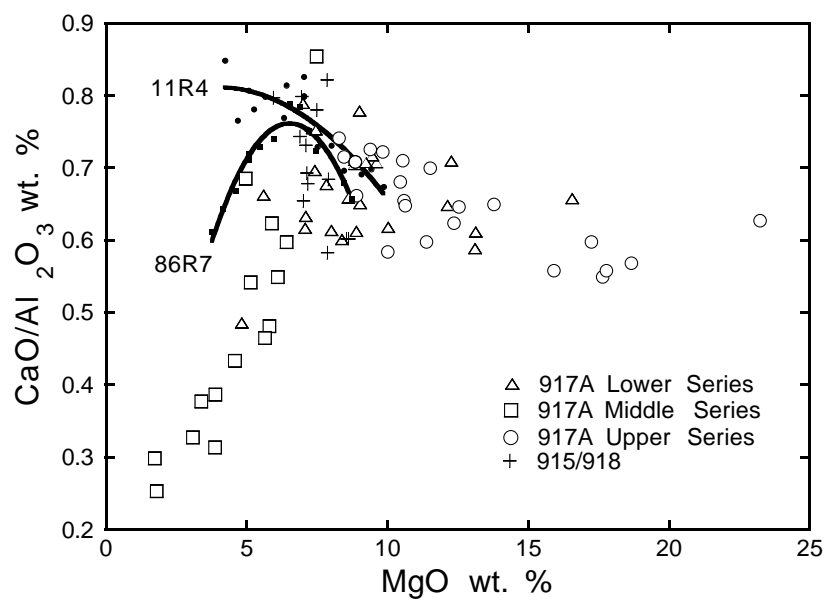

Figure 5. $\mathrm{MgO}$ vs. $\mathrm{CaO} / \mathrm{Al}_{2} \mathrm{O}_{3}(\mathrm{wt} \%)$ for the experimental glasses and $\mathrm{Leg}$ 152 whole rock samples. Data and symbols as for Figure 4. tance of low pressure phase equilibria for evolution of Leg 152 magmas.

The most striking observation in Figure 4 is that the compositional trends for the most evolved lavas ( $<6 \mathrm{wt} \% \mathrm{MgO})$ do not agree well with the experimentally determined LLD. The majority of the differentiated lavas at Site 917 have higher $\mathrm{SiO}_{2}, \mathrm{Al}_{2} \mathrm{O}_{3}$, and $\mathrm{Na}_{2} \mathrm{O}$, and lower $\mathrm{TiO}_{2}, \mathrm{FeO}$, and $\mathrm{CaO}$ than the experimental cotectic liquids. These lavas are primarily from the Middle Series and were interpreted by the Shipboard Scientific Party to have assimilated significant amounts of crustal material (Larsen, Saunders, Clift, et al., 1994). However, there are also a few units with substantially higher $\mathrm{FeO}$, $\mathrm{TiO}_{2}$, and $\mathrm{P}_{2} \mathrm{O}_{5}$ contents that approach values characteristic of the plateau lavas of the Scoresby Sund area (Larsen et al., 1989). These units include the sill from Site 918 and a few units from the Lower and Middle Series at Site 917. In these cases, correspondence with the experimental LLD is good and suggests that these magmas escaped contamination. Between $6 \%$ and $10 \% \mathrm{MgO}$, the compositional trends for Leg 152 lavas more closely resemble the experimental LLD.

The four stratigraphic groups (Lower, Middle, and Upper Series at Site 917 and the oceanic series at Sites 915 and 918) each show a different relationship between $\mathrm{MgO}$ and $\mathrm{Al}_{2} \mathrm{O}_{3}$. Only the variations displayed by the Upper Series of Site 917 and Sites 915/918 are ex-
A.

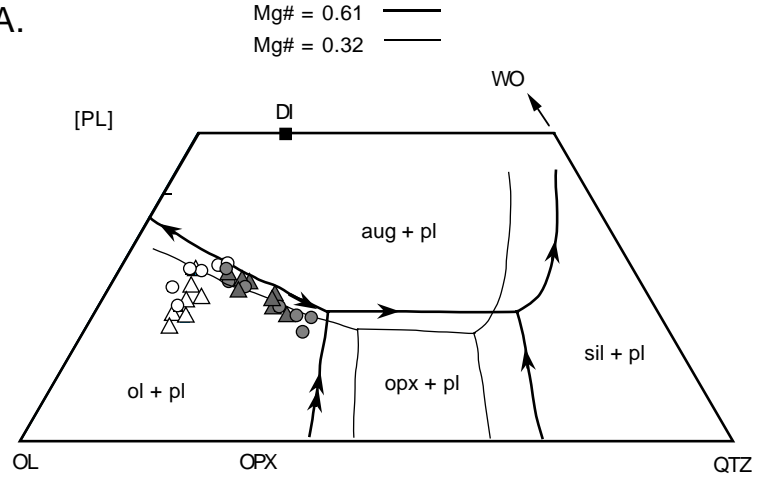

O 11R4 experimental liquids

$\triangle 86 \mathrm{R} 7$ experimental liquids

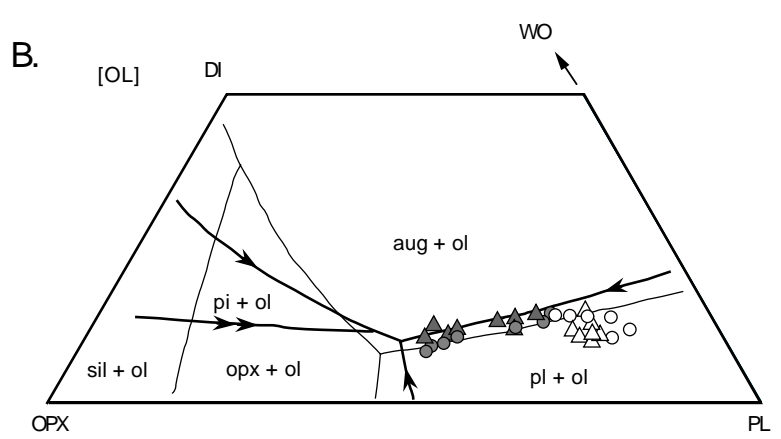

Open symbols: ol or ol+pl saturated Filled symbols: $\mathrm{pl}+\mathrm{ol}+\mathrm{cpx}$ saturated
C.
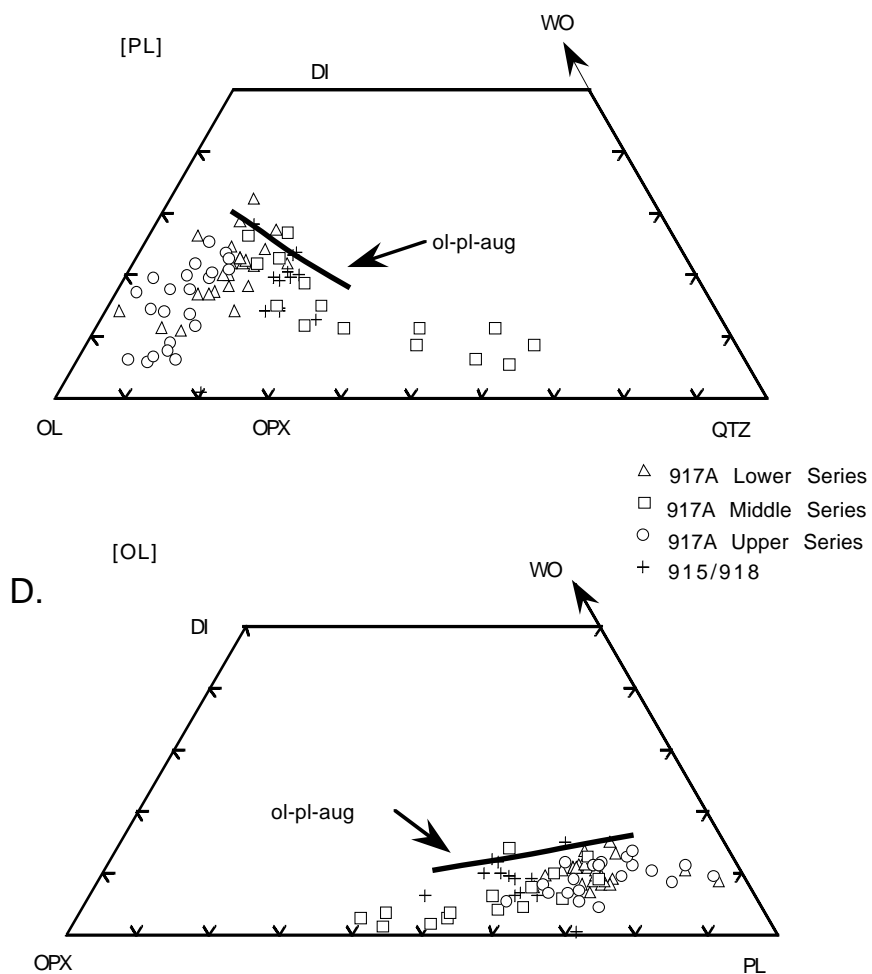

Figure 6. Experimental results portrayed in the oxygen unit projection scheme of Longhi (1991) projected from plagioclase (A) and olivine (B). Heavy and light lines show the positions of predicted low pressure phase boundaries for the most primitive $(\mathrm{Mg} \#=0.61)$ and most differentiated $(\mathrm{Mg} \#=0.32)$ experimental glasses using algorithms presented by Longhi (1991, appendix 1). Figures $\mathbf{C}$ and $\mathbf{D}$ show the compositions of Leg 152 igneous units compared to the experimental olivine-plagioclase-augite cotectic (heavy lines) projected from plagioclase (C) and olivine (D). Symbols for Leg 152 data are the same as for Figure 4. Abbreviations for this and following projections are for the normative components olivine (OL), quartz (QTZ), wollastonite (WO), diopside (DI), plagioclase (PL), orthopyroxene (OPX), and for mineral phases olivine (ol), plagioclase (pl), orthopyroxene (opx), $\mathrm{SiO}_{2}$ minerals (sil), augite (aug), pigeonite (pi). Experimental olivine and plagioclase compositions project near the olivine and plagioclase projection coordinates, respectively, while experimental augite compositions are displaced from the projected position of pure DI due to the presence of non-quadrilateral components in the augites. The projection equations in oxygen units for the plagioclase projection $[\mathrm{PL}]$ from Longhi (1991) are: $\mathrm{Ol}[\mathrm{Pl}]=2\left(\mathrm{FeO}+\mathrm{MgO}+\mathrm{MnO}+2 \mathrm{Fe}_{2} \mathrm{O}_{3}\right) / \mathrm{Sum}$; Wo[Pl] $=3\left(\mathrm{CaO}-\mathrm{Al}_{2} \mathrm{O}_{3}+\mathrm{K}_{2} \mathrm{O}+\right.$ $\left.\mathrm{Na}_{2} \mathrm{O}\right) / \mathrm{Sum} ; \mathrm{Qtz}[\mathrm{Pl}]=\left(2 \mathrm{SiO}_{2}-\mathrm{FeO}+\mathrm{MgO}+\mathrm{MnO}+2 \mathrm{Fe}_{2} \mathrm{O}_{3}-2 \mathrm{Al}_{2} \mathrm{O}_{3}-2 \mathrm{CaO}-10\left[\mathrm{~K}_{2} \mathrm{O}+\mathrm{Na}_{2} \mathrm{O}\right]\right) / \mathrm{Sum}$. For the olivine projection $[\mathrm{OL}]: \mathrm{Opx}[\mathrm{Ol}]=3\left(2 \mathrm{SiO}{ }_{2}\right.$ $\left.-\mathrm{FeO}+\mathrm{MgO}+\mathrm{MnO}+2 \mathrm{Fe}_{2} \mathrm{O}_{3}-2 \mathrm{CaO}-2 \mathrm{Al}_{2} \mathrm{O}_{3}-10\left[\mathrm{~K}_{2} \mathrm{O}+\mathrm{Na}_{2} \mathrm{O}\right]\right) / \mathrm{Sum} ; \mathrm{Wo}[\mathrm{Ol}]=3\left(\mathrm{CaO}-\mathrm{Al}_{2} \mathrm{O}_{3}+\mathrm{K}_{2} \mathrm{O}+\mathrm{Na}_{2} \mathrm{O}\right) / \mathrm{Sum} ; \mathrm{Pl}[\mathrm{Ol}]=\left(8 \mathrm{Al} \mathrm{O}_{3}+8 \mathrm{Na}_{2} \mathrm{O}-\right.$ $\left.8 \mathrm{~K}_{2} \mathrm{O}\right) / \mathrm{Sum}$. For both projections, "Sum" is the summation of the three equations. The input oxides are in mole units. 
plicable in terms of the low pressure experimental liquid lines of descent. In the Upper Series, $\mathrm{Al}_{2} \mathrm{O}_{3}$ steadily increases as $\mathrm{MgO}$ decreases, suggesting crystallization of olivine alone. This is consistent with the petrography indicating that olivine is the only phenocryst phase (Larsen, Saunders, Clift, et al., 1994). The LLDs determined for $11 \mathrm{R} 4$ and $86 \mathrm{R} 7$ show a brief interval of increasing $\mathrm{Al}_{2} \mathrm{O}_{3}$ with decreasing $\mathrm{MgO}$. However at $\sim 8 \mathrm{wt} \% \mathrm{MgO}$, plagioclase begins to crystallize, and the $\mathrm{Al}_{2} \mathrm{O}_{3}$ content of the liquid decreases along with $\mathrm{MgO}$. Because the most fractionated Upper Series samples have compositions of $\sim 9 \mathrm{wt} \% \mathrm{MgO}$, this portion of the LLD is not represented in the Site 917 Upper Series, but it is closely followed by the more differentiated lavas from Sites 915 and 918. The variations of $\mathrm{Al}_{2} \mathrm{O}_{3}$ with $\mathrm{MgO}$ for the Lower and Middle Series at Site 917, however, are markedly different from the experimental LLDs. For example, the differentiated Lower Series generally have higher $\mathrm{Al}_{2} \mathrm{O}_{3}$ contents compared to $86 \mathrm{R} 7$ experimental liquids at similar $\mathrm{MgO}$ content. The same is also found for the Middle Series, although direct comparison with LLDs for the Upper and Lower Series is admittedly tenuous. Nevertheless, what appears clear is that the elevated $\mathrm{Al}_{2} \mathrm{O}_{3}$ contents of the more differentiated members of the Lower and Middle Series cannot be readily explained solely in terms of the low pressure phase relations.

Additional insights into the nature of the fractionating mineral assemblage is provided by considering the location of the olivine-plagioclase-augite cotectic in relation to the compositions of erupted lavas (Fig. 6C, D). With few exceptions, Leg 152 lavas plot below the multisaturated cotectic within the phase volumes defined for olivine and olivine + plagioclase. Based on the projected positions relative to the olivine-plagioclase-augite cotectic, we can infer that the majority of the recovered flows evolved at low pressure by olivine and plagioclase crystallization and that few reached augite saturation. These conclusions are consistent with the crystallization sequence olivine, plagioclase, and followed by augite deduced from shipboard petrography for Site 917 Upper and Lower Series lavas (Larsen, Saunders, Clift, et al., 1994). The evolved lavas from the Middle Series form distinct arrays in the plagioclase and olivine projections (Fig. 6C, D) that are subparallel and displaced from the low pressure multisaturated cotectic trending toward the quartz and orthopyroxene apices, respectively. These trends are clearly unrelated to the experimental LLD, but are consistent with contamination by continental crust (Larsen, Saunders, Clift, et al., 1994). This observation is consistent with trace element data of Fitton et al. (Chap. 28, this volume) showing high $\mathrm{Ba} / \mathrm{Zr}$ ratios, suggestive of significant amounts of contamination, in the Lower and Middle Series at Site 917. In contrast, the Upper Series at Site 917 and the Site 918 flows show relatively low $\mathrm{Ba} / \mathrm{Zr}$ ratios suggesting they escaped significant crustal contamination, which is consistent with conclusions reached from the experimental data.

The importance of olivine and plagioclase crystallization for the more primitive members of the Lower and Upper Series can be examined more closely in a projection from wollastonite onto the olivine-plagioclase-quartz plane showing the locations of the olivine, plagioclase, and low-Ca pyroxene fields (Longhi, 1991). Figure 7 illustrates the topologies appropriate for various liquid compositions, including the experimental starting compositions. The main ternary projection of Figure 7 shows all the primary phase volumes specific to the compositional parameters of Sample 11R4. Also plotted in this projection is the position of the olivine-plagioclase cotectic predicted for three other samples (curves 2-4) that are shown individually as companion plots. Figure 7 shows that the Upper Series lavas fall to the left of the 11R4 olivine-plagioclase cotectic (curve 1) well within the olivine field. Although this is simply another representation of the relationships shown in Figure 4, it confirms the importance of olivine control on the composition of these lavas. In contrast, the Middle Series lavas project into the plagioclase field and form a trend toward increasing quartz component. This trend is subparallel to a simple mixing array involving the sample of Archean basement from the coastal region of southeast Greenland (Blichert-Toft et al., 1995), suggested by Fitton et al. (Chap. 28, this volume) as a possible contaminant.

The relationships displayed by the Lower Series lavas are more puzzling. The lavas project on both sides of the olivine-plagioclase cotectic defined for $86 \mathrm{R} 7$ (curve 2; Figure 7). We are not troubled by the subset of lavas projecting into the olivine field or on the cotectic, as their plotted locations are consistent with both the low pressure experimental relations and shipboard petrography. However, those lavas projecting into the plagioclase field imply that plagioclase is the liquidus phase at low pressure. This expectation is inconsistent with the compositional relationship portrayed in Figure 4, as well as shipboard petrography that reported either olivine or olivine + plagioclase as microphenocrysts but not plagioclase alone (Larsen, Saunders, Clift, et al., 1994). What have not been accounted for in this assessment of the projected compositional relations are the effects of composition, specifically $\mathrm{Mg \#}$ and alkali contents, on the position of the olivine-plagioclase cotectic. For example, the olivine-plagioclase cotectic for 86R7, compared with 11R4, is slightly displaced toward plagioclase due to higher albite (NAB) and orthoclase (NOR) components of the normative feldspar (Fig. 7). More extreme movement of the olivine-plagioclase cotectic is found for a number of the more differentiated Lower Series lavas. For example, the phase boundaries for Units 71 and 72 (curves 3 and 4) are shifted appreciably in the direction of the quartz-plagioclase join. It is also pertinent that for both these units lava composition coincides with the predicted olivine-plagioclase cotectic. Viewed in this way, Units 71 and 72 are not anomalous in their elevated normative plagioclase content. The expectation that these lavas should be co-saturated with olivine and plagioclase at low pressure is consistent with the observed groundmass assemblage of olivine and plagioclase.

Given the compositional control on the position of the phase boundaries, it becomes necessary to consider the cotectic relations for individual lavas. This exercise has been performed for each of the Lower Series lavas with the result that with the exception of five units $(76,79,82,89$, and 91), all plot either on or to the left of their pertinent olivine-plagioclase cotectic. The fact that a large number of the Lower Series lavas are consistent with the one-atmosphere phase relations, shown experimentally in this study and predicted using the model of Longhi (1991), supports the view that differentiation of the Lower Series was controlled primarily by low pressure phase relations. The exceptions noted above are important because they point to more complicated controls on differentiation.

Figures 4 and 8 suggest that the high $\mathrm{Al}_{2} \mathrm{O}_{3}$ and normative plagioclase contents of Units $76,79,82,89$, and 91 are due to a greater interval of olivine crystallization than expected at low pressure. Part of the expansion of the olivine primary phase volume relative to plagioclase may be related to addition of alkalis. Figure 8 shows the shift of the cotectic after addition of up to $20 \mathrm{wt} \%$ Archean basement. Such large amounts of contamination would yield compositions quite distinct from the "high-Al" lavas under consideration, and the predicted shift in the olivine-plagioclase cotectic is actually very small and hence does not change the topology of the system appreciably.

A number of other factors can increase the interval of olivine crystallization and suppress plagioclase appearance. Both increased lithostatic and increased water pressure act to destabilize plagioclase. Anhydrous pressure has been shown to have modest effect on the position of the olivine-plagioclase cotectic, but it greatly expands the primary phase volume for pyroxene relative to both olivine and plagioclase (Presnall et al., 1978; Fram and Longhi, 1992; Grove et al., 1992). No evidence is found for early augite crystallization. This is in contrast to the lower lavas of the East Greenland flood province to the north where augite appears as an early crystallizing phenocryst (Fram and Lesher, 1997). Alternatively, elevated water contents are also known to cause contraction of the plagioclase phase volume, while having comparatively little effect on the relative stabilities of olivine and augite (Kushiro, 1972; Thompson et al., 1972; Baker and Eggler, 1983, 1987; Spulber and Rutherford, 1983; Merzbacher and Eggler, 1984; Grove and Kinzler, 1986; Beard and Lofgren, 1992; Sisson and 


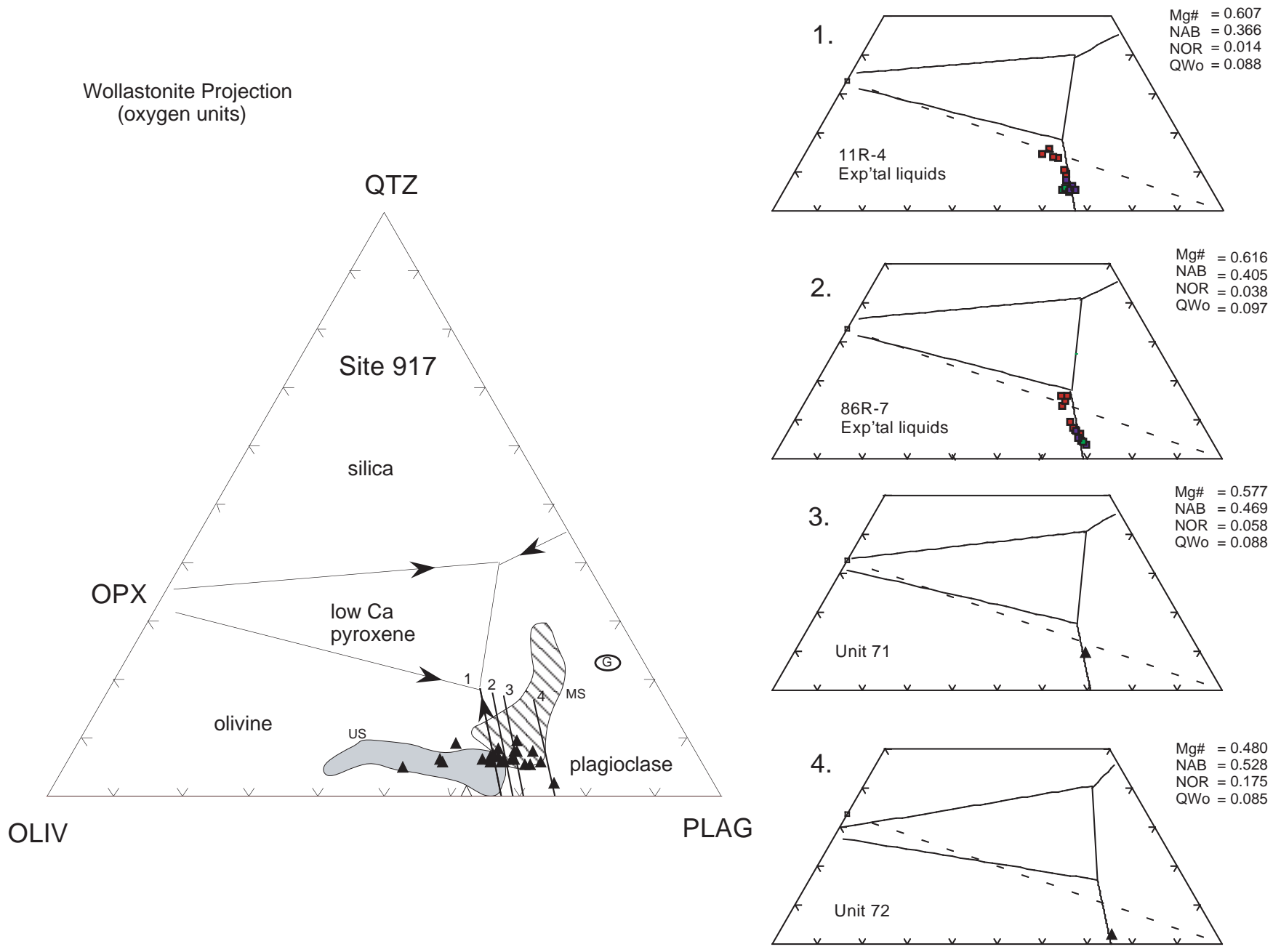

Figure 7. Experimental and predicted phase boundaries projected in oxygen units from wollastonite using the projection components of Longhi (1991). The shaded field includes all Upper Series units from Site 917 (US), and the hatched field encompasses the Middle Series (MS). Solid triangles = Lower Series units. The composition labeled " $G$ " is for Archean basement reported by Blichert-Toft et al. (1995; analysis 324721) and suggested as a possible crustal contaminant by Fitton et al. (Chap. 28, this volume). The accompanying plots labeled 1-4 show the positions of the calculated phase boundaries using the parameterizations of Longhi (1991) for systems with values of Mg\#, NAB, NOR, and QWo equal to four Site 917 lava compositions. Models 1 and 2 are for samples 11R4 and 86R7. Models 3 and 4 are for Units 71 and 72, respectively. The predicted olivine-plagioclase cotectics are compared on the main figure and annotated by 1,2 , 3 , and 4, respectively. The variables given for each prediction model are in oxide mole units where: $\mathrm{Mg} \#=\mathrm{MgO} /(\mathrm{MgO}+\mathrm{FeO}) ; \mathrm{NAB}=2 \mathrm{Na}_{2} \mathrm{O} /\left(\mathrm{Al}_{2} \mathrm{O}_{3}+\mathrm{Na}_{2} \mathrm{O}\right.$ $+\mathrm{K}_{2} \mathrm{O}$ ) (albite fraction in normative plagioclase); $\mathrm{NOR}=2 \mathrm{~K}_{2} \mathrm{O} /\left(\mathrm{Al}_{2} \mathrm{O}_{3}+\mathrm{Na}_{2} \mathrm{O}+\mathrm{K}_{2} \mathrm{O}\right)$ (orthoclase fraction in normative plagioclase); QWo $=$ quaternary wollastonite component (see, Longhi, 1991). The equations in oxygen units for the wollastonite projection $[\mathrm{WO}]$ are: $\mathrm{Ol}[\mathrm{WO}]=2\left(\mathrm{FeO}+\mathrm{MgO}+\mathrm{MnO}+2 \mathrm{Fe}_{2} \mathrm{O}_{3}\right) /$ $\left.\mathrm{Sum} ; \mathrm{Qtz}[\mathrm{WO}]=\left(2 \mathrm{SiO}_{2}-\mathrm{FeO}+\mathrm{MgO}+\mathrm{MnO}+2 \mathrm{Fe}_{2} \mathrm{O}_{3}\right)-2 \mathrm{Al}_{2} \mathrm{O}_{3}-2 \mathrm{CaO}-10\left[\mathrm{~K}_{2} \mathrm{O}+\mathrm{Na}_{2} \mathrm{O}\right]\right) / \mathrm{Sum} ; \mathrm{Pl}[\mathrm{Wo}]=\left(8 \mathrm{Al}_{2} \mathrm{O}_{3}+\mathrm{Na}_{2} \mathrm{O}-\mathrm{K}_{2} \mathrm{O}\right) / \mathrm{Sum}$. $\mathrm{Sum}$ " is the summation of the three equations. Oxides in mole units.

Grove, 1993; Gaetani et al., 1993). Although compositional data for hydrous basaltic melts in equilibrium with olivine and plagioclase are limited, the hydrous melting experiments by Spulber and Rutherford (1983) provide some estimate of the magnitude of the effect on the projected location of the olivine-plagioclase cotectic.

Figure 8 shows the location of the olivine-plagioclase cotectic for a Galapagos basalt at $\mathrm{f}_{\mathrm{H} 2 \mathrm{O}}=0.67 \mathrm{~kb}$ and $\mathrm{P}_{\text {total }}=1 \mathrm{~kb}$ (Spulber and $\mathrm{Ru}-$ therford, 1983). Anhydrous experiments were not reported for this composition; however, the low pressure olivine-plagioclase cotectic predicted using the model of Longhi (1991) corresponds closely to our experimental cotectics for 11R4 and 86R7. As seen in Figure 8, the shift in the olivine-plagioclase cotectic with addition of water is substantial and far exceeds that required to place the "high-Al" lavas within the olivine field. On the other hand, more modest water contents could readily account for the protracted interval of olivine crystallization that appears necessary to explain the composition of the "high-Al" lavas of the Lower Series. We consider the most likely source of this water to be introduced during assimilation of crustal material, which has variably modified many of the differentiated members of the suite. However, we cannot preclude the possibility that the inferred hydrous character of these magmas is more directly linked to mantle source and melting conditions. Further experimental work to characterize the hydrous phase relations at elevated pressures is clearly needed to verify our working hypothesis.

\section{Origin of the Picritic Flows and Estimated Primary Melt Compositions}

The compositional data and petrography discussed in the preceding section demonstrate that many of the primitive aphyric lavas of the Upper and Lower Series are the product of variable amounts of olivine fractionation prior to eruption. Thus, only a small group of flows with relatively high magnesium contents may represent primary liquid compositions. Although many of these lavas are classified as picrites (>15\% modal olivine), it is necessary to establish case by case whether the bulk composition of any lava is a permissible liquid 
Figure 8. Part of the wollastonite projection of Figure 7 showing the predicted low pressure anhydrous olivinephase boundary for Units 76, 79, 82, 89, and 91 (triangles). Note that these "high-Al" lavas plot to the right of olivine-plagioclase within the plagioclase field. The effect of adding $20 \%$ contaminant represented by " $G$ " on the position of the anhydrous cotectic is shown by the light dashed curve. The location of the "wet" olivine-plagioclase cotectic at $\mathrm{f}_{\mathrm{H} 2 \mathrm{O}}=0.67 \mathrm{~kb}$ and $\mathrm{P}_{\text {total }}=1 \mathrm{~kb}$ for a Galapagos basalt is shown as the heavy dashed curve (Spulber and Rutherford, 1983).

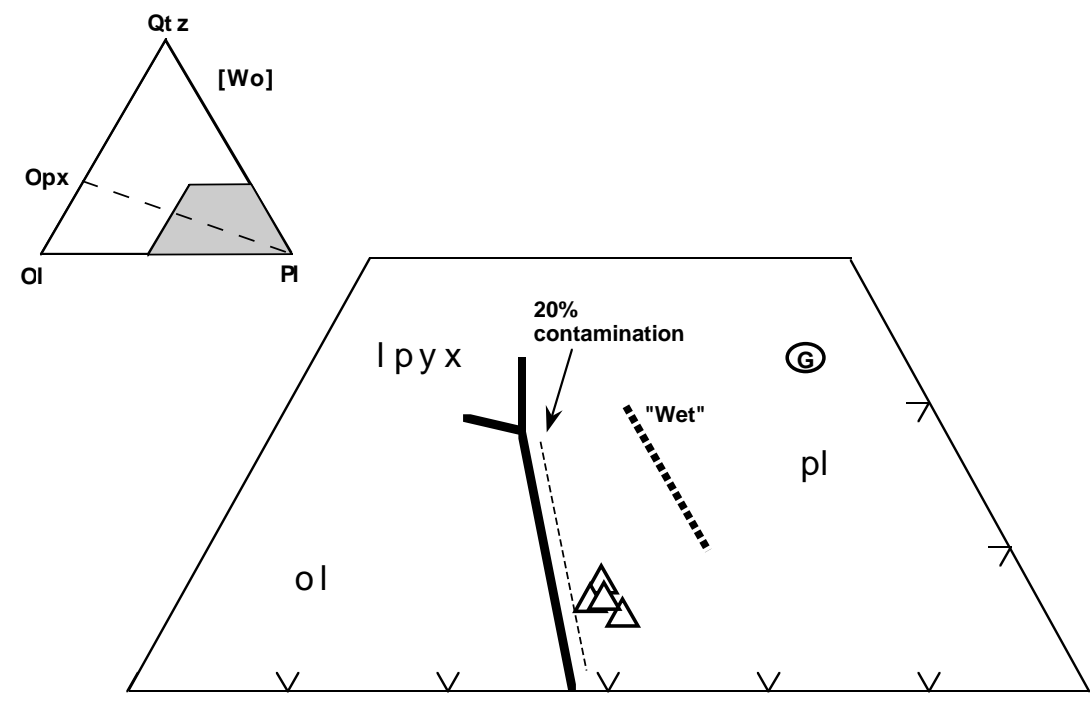

composition or whether the composition was modified by olivine accumulation. The suitability as a liquid composition can be evaluated by examining the covariations of $\mathrm{FeO}$ and $\mathrm{MgO}$ in more detail (Fig. 9). For reference, the LLD for the Upper and Lower Series have been extrapolated to higher $\mathrm{MgO}$ content by adding olivine incrementally in steps of $1 \%$ of the equilibrium olivine by weight beginning with the compositions of the glasses produced in runs 11R4-15 and 86R715. The LLD calculated in this way are shown in Figure 9 with the composition of the equilibrium olivine noted above $\mathrm{Fo}_{90}$. These calculations are referenced to the low pressure conditions of this study, but they will not be altered significantly by employing possible pressure dependencies on the $K_{\mathrm{D}} \mathrm{s}$ and redox conditions below the FMQ oxygen buffer.

It is noteworthy that, with few exceptions, the picritic lavas described by the Shipboard Scientific Party as highly olivine phyric generally plot above the back-calculated LLD for olivine fractionation. The modes of phenocrystic olivine reported by Larsen, Saunders, Clift, et al. (1994) are indicated in parentheses and range from $10 \%$ to $48 \%$ olivine by volume. Most of the variation seen in the picritic lavas can be explained by accumulation of up to $25 \%$ olivine $\left(\mathrm{Fo}_{84-85}\right)$, which is in equilibrium with liquids with $8-10 \mathrm{wt} \% \mathrm{MgO}$. Likewise, it is inconceivable that the most magnesian picrites containing 43\%-48\% modal olivine (Fig. 9) could represent liquids in equilibrium with any reasonable mantle olivine. We conclude from these considerations that most of the lavas classified as picrite do not represent reliable primary liquid compositions.

There are important exceptions among the high magnesian lavas, and these are represented by the aphyric flows reported from the Lower and Upper Series (Larsen, Saunders, Clift, et al., 1994). Figure 9 shows that these flows are the only whole rock compositions lying on or close to the pertinent LLD. These flows include Units 14 and 16 from the Upper Series and Unit 78, and possibly Unit 62, from the Lower Series (Larsen et al., Chap. 27, this volume). For Units 14 and 16 the equilibrium olivine is predicted to be $\mathrm{Fo}_{90-91}$, supporting the contention that these lavas are unfractionated mantle melts. Demant et al. (this volume) report olivine compositions up to $\mathrm{Fo}_{92.5-90.7}$ in accumulative picrites from the Upper and Lower Series. Some of these olivine compositions are slightly higher than those estimated to be equilibrium with the aphyric high-magnesian flows at oxygen fugacities of the FMQ buffer but may be entirely consistent with higher oxygen fugacities.

A more complete comparison of the compositions of the primitive aphyric flows and calculated liquids in equilibrium with mantle olivine is provided in Table 7. The compositions of Unit 16 and the backcorrected primary liquid for 11R4 are in good agreement. Unit 14 is also similar, but has slightly lower $\mathrm{TiO}_{2}$ and $\mathrm{CaO}$, and higher $\mathrm{Al}_{2} \mathrm{O}_{3}$. For further comparison, the composition of Unit 17 also has been back-corrected for olivine fractionation, and yields an estimate of a primary mantle melt having higher $\mathrm{TiO}_{2}$ and $\mathrm{Na}_{2} \mathrm{O}$, and lower $\mathrm{Al}_{2} \mathrm{O}_{3}$ compared to Units 14 and 16 . Similar comparisons cannot easily be made among members of the Lower Series; however, the back-corrected composition for $86 \mathrm{R} 7$ is clearly different than the compositions estimated for the Upper Series, not only in terms of $\mathrm{FeO}$ and $\mathrm{MgO}$, but also $\mathrm{CaO}, \mathrm{Al}_{2} \mathrm{O}_{3}, \mathrm{Na}_{2} \mathrm{O}$, and $\mathrm{K}_{2} \mathrm{O}$ contents.

The results summarized in Figure 9 and Table 7 suggest fairly uniform composition for calculated primary melts and primitive Upper Series flows all with $\mathrm{MgO}$ of $\sim 18 \mathrm{wt} \%, \mathrm{FeO}_{\text {total }}$ of $\sim 11 \mathrm{wt} \%$, and $\mathrm{TiO}_{2}$ of $\sim 1 \mathrm{wt} \%$ (11R4, Units $14,16,17)$. In contrast, the primary melt estimated from $86 \mathrm{R} 7$ for the Lower Series has lower $\mathrm{FeO}_{\text {total }}(\sim 9.7 \mathrm{wt} \%)$ and $\mathrm{MgO}$, and high $\mathrm{CaO}, \mathrm{Al}_{2} \mathrm{O}_{3}$, and, possibly, $\mathrm{Na}_{2} \mathrm{O}$.

Estimates of the liquidus temperatures for this suite of primary melts are also provided in Table 7. An estimated 1-atm liquidus temperature for the primary Upper Series melt is $\sim 1380^{\circ} \mathrm{C}$, while the temperature for the proposed primary liquid for the Lower Series is $\sim 1340^{\circ} \mathrm{C}$. Although these temperatures are hypothetical for the backcorrected compositions, the estimates for Units 14 and 16 do represent reasonable indications of eruption temperature. The temperature estimated for the Lower Series also should be regarded as a maximum value, as the addition of small amounts of water will significantly lower the liquidus temperature. Although these temperatures are not direct estimates of mantle potential temperature, they do suggest an increase in the potential temperature of the source region for basalts with time at Site 917. A more rigorous assessment of the thermal state of the mantle during transient development of the continental succession, however, requires independent estimates of the mean pressure and extent of melting (see Fram et al., this volume).

Recent efforts to understand the compositions of mid-ocean-ridge basalts (MORB) and the systematics of mantle melting have highlighted the relationships between extent and pressure of mantle melting and the composition of primary liquids (e.g., Langmuir et al., 1992). Of importance here is the observation that the mean FeO content of mantle melts increases with mean pressure of melting. For MORBs the mean pressure has been shown to correlate positively with mean extent of melting. Klein and Langmuir (1989) related these variations globally to changes in the thermal structure of upwelling mantle beneath ridges. Fram and Lesher (1993) considered the effect of imposing a barrier to mantle upwelling during the early stages of continental rifting and developed a model to explain magmatic variations in the North Atlantic basin during the transition from continental to oceanic magmatism. Their model predicts that during 


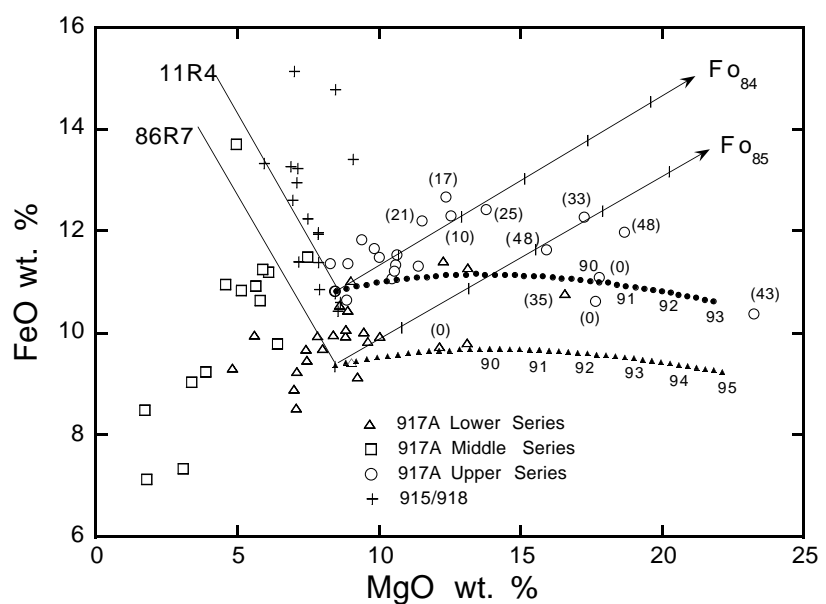

Figure 9. Compositional variation in $\mathrm{FeO}-\mathrm{MgO}$ of lavas from Leg 152. Solid lines are the liquid lines of descent for 11R4 and 86R7. Solid arrows indicate the effect of accumulation of equilibrium olivine $\left(\mathrm{Fo}_{84}\right.$ and $\left.\mathrm{Fo}_{85}\right)$ in liquid compositions saturated in olivine (11R4-15 and 86R7-15). Each tick indicates $5 \%$ olivine accumulation. The array of solid symbols represents LLDs for perfect olivine fractionation computed by incrementally adding equilibrium olivine in steps of $1 \%$ olivine by weight to experimental liquids $11 \mathrm{R} 4$ 15 and 86R7-15. The values plotted along these curves represent the forsterite content of liquidus olivine for the FMQ oxygen buffer. The modal proportion of phenocrystic olivine reported by Larsen, Saunders, Clift, et al. (1994) for samples with $>10 \mathrm{wt} \% \mathrm{MgO}$ is given in parentheses. Aphyric flow is indicated by " 0 ." Note that modal data are not available for a number of magnesian lavas.

the early stages of rift magmatism, primary melts will have higher $\mathrm{FeO}$ and incompatible element contents due to modest extents of partial melting at high mean pressures beneath the continental lithosphere. Thinning of the lithosphere permits mantle upwelling to shallow depths resulting in a decrease in the mean pressure and increase in the mean extent of melting. Pooled mantle melts formed at the onset of seafloor spreading are then characterized by lower $\mathrm{FeO}$ and incompatible element contents, and approach the inferred compositions for MORBs. Fram and Lesher (1993) showed a strong positive correlation between $\mathrm{FeO}$ and $\mathrm{TiO}_{2}$ contents of estimated primary melts erupted over the last $60 \mathrm{~m} . y$. ., where the earliest rifted products defined the high end of the range and North Atlantic MORB and Iceland constrained the low end.

Application of these concepts to the magmatic development of the southeast Greenland volcanic margin is explored by Fram et al. (this volume). However, it is interesting to note that the temporal variations in primary melt composition inferred at Site 917 are not consistent with a simple "lid" effect controlling the overall extent and depth of melting. Rather the primary liquids for the Lower Series are lower in $\mathrm{FeO}$ and $\mathrm{TiO}_{2}$ compared to the younger, Upper Series (Table 7). It also should be noted that the $\mathrm{FeO}$ and $\mathrm{TiO}_{2}$ contents for primary melts estimated for the Upper Series are similar to those for the Hatton Bank and Scoresby Sund (Fram and Lesher, 1993), while the Lower Series approach the range for MORB. A factor that may be important during the very early stages of continental magmatism is the influence of lithospheric structure on melt segregation and focusing within the region of upwelling. Langmuir et al. (1992) considered consequences of incomplete focusing and suggested that poor delivery of melts to the spreading zone can lead to preferential tapping of melts formed at the highest levels of the melting column. These melts, in general, will have lower $\mathrm{FeO}$ and will be depleted in incompatible elements. During lithospheric thinning and asthenospheric upwelling, increased continuity within the mantle source with time will permit segregation of lower degree melts, higher in $\mathrm{FeO}$ and $\mathrm{TiO}_{2}$, from greater depth. The expectation is that $\mathrm{FeO}$ and $\mathrm{TiO}_{2}$ contents of primary melts should increase with time, consistent with the observa-
Table 7. Primary magma compositions.

\begin{tabular}{|c|c|c|c|c|c|}
\hline & $11 \mathrm{R} 4$ & $86 \mathrm{R} 7$ & Unit 17 & \multirow[b]{2}{*}{ Unit 14} & \multirow[b]{2}{*}{ Unit 16} \\
\hline & \multicolumn{3}{|c|}{ Estimated } & & \\
\hline $\mathrm{SiO}_{2}$ & 46.88 & 47.81 & 47.08 & 46.77 & 47.38 \\
\hline $\mathrm{TiO}_{2}$ & 0.96 & 0.90 & 1.12 & 0.72 & 0.91 \\
\hline $\mathrm{Al}_{2} \mathrm{O}_{3}$ & 12.40 & 13.93 & 11.92 & 14.30 & 13.09 \\
\hline $\mathrm{Fe}_{2}^{2} \mathrm{O}_{3}$ & 1.45 & 1.32 & 1.49 & 1.26 & 1.30 \\
\hline $\mathrm{FeO}$ & 9.70 & 8.49 & 9.70 & 9.51 & 9.92 \\
\hline $\mathrm{FeO}^{*}$ & 11.00 & 9.68 & 11.05 & 10.65 & 11.09 \\
\hline $\mathrm{MnO}$ & 0.19 & 0.16 & 0.18 & 0.20 & 0.24 \\
\hline $\mathrm{MgO}$ & 17.76 & 15.31 & 17.76 & 17.67 & 17.80 \\
\hline $\mathrm{CaO}$ & 8.71 & 9.52 & 8.60 & 7.86 & 7.31 \\
\hline $\mathrm{Na}_{2} \mathrm{O}$ & 1.67 & 2.12 & 1.94 & 1.61 & 1.78 \\
\hline $\mathrm{K}_{2} \mathrm{O}$ & 0.17 & 0.31 & 0.13 & 0.06 & 0.23 \\
\hline $\mathrm{P}_{2} \mathrm{O}_{5}$ & 0.11 & 0.13 & 0.08 & 0.02 & 0.04 \\
\hline \multicolumn{6}{|l|}{ Liquids } \\
\hline Temp. $\left({ }^{\circ} \mathrm{C}\right)^{\dagger}$ & 1378 & 1337 & 1378 & 1375 & 1377 \\
\hline $\mathrm{Mg} /\left(\mathrm{Mg}+\mathrm{Fe}^{2+}\right)$ & 0.766 & 0.763 & 0.765 & 0.768 & 0.762 \\
\hline Fo (FMQ) & 91 & 91 & 91 & 91 & 91 \\
\hline Fo (FMQ-3) & 90 & 90 & 90 & 90 & 90 \\
\hline
\end{tabular}

Notes: The compositions are estimated by incrementally adding olivine to the glasses produced in runs 11R4-15 and 86R7-15 and to the analysis of Unit 17 (Larsen et al., Chap. 27, this volume). Units 14 and 16 are from Larsen et al. (Chap. 27, this volume) and have not been back-calculated to higher $\mathrm{MgO}$ contents. The oxides have been normalized to a total of 100 , and $\mathrm{Fe}^{3+} / \mathrm{Fe}^{2+}$ adjusted to the forsterite-magnetite-quartz oxygen buffer (FMQ). $\mathrm{FeO}^{*}$ is all iron calculated as $\mathrm{FeO}$. Fo is composition of equilibrium olivine as percent forsterite for the FMQ oxygen buffer and three $\log$ units below (FMQ-3). $\dagger=$ estimated liquidus eruption temperature (see text for discussion) calculated by extending the $\mathrm{T}-\mathrm{MgO}$ relationships to higher $\mathrm{MgO}$ contents and including pure forsterite. The equation used is based on Thy (1995): $\mathrm{T}\left({ }^{\circ} \mathrm{C}\right)=\mathrm{a}+\exp \left(\mathrm{b} \cdot \mathrm{x}+\mathrm{c} \cdot \mathrm{x}^{2}+\mathrm{d} \cdot \mathrm{x}^{3}+\mathrm{e} \cdot \mathrm{x}^{4}\right)$, where $\mathrm{x}$ is $\mathrm{MgO}$ in wt $\%, \mathrm{a}=1005, \mathrm{~b}=$ $2.64 \cdot 10^{-2}, \mathrm{c}=-6.60 \cdot 10^{-4}, \mathrm{~d}=1.09 \cdot 10^{-5}, \mathrm{e}=-7.2 \cdot 10^{-8}$.

tions for Site 917 . This model, however, does not readily account for (1) the hiatus in volcanism indicated by deposition of the sediments after emplacement of the Middle Series, and (2) the massive outpouring of primitive basalts represented by the Upper Series. This stratigraphic relation suggests an increase in the rates of extension and melt production with eruption of the Upper Series lavas. It is possible that eruption of the Upper Series signals the approach of a proto-Iceland plume in the region and may argue for a changing mantle source with time (Larsen, Saunders, Clift, et al., 1994; Fitton et al, Chap. 28, this volume; Fram et al., this volume). Fram et al. (this volume) have shown that the uppermost parts of Hole 917A are characterized by interfingering of two compositional distinct lava groups. A group with low $\mathrm{La} / \mathrm{Sm}_{\mathrm{N}}$ is inferred to be related to a normal mid-ocean basalt source, while a group with high $\mathrm{La} / \mathrm{Sm}_{\mathrm{N}}$ is suggested to be related to the arrival of a depleted Icelandic mantle source.

Finally, our interpretation that primitive melts of the Lower Series were hydrous opens the possibility that mantle melting initially occurred under damp conditions. If so, small amounts of water would have significantly lowered solidus temperatures and could have resulted in enhanced melting of asthenospheric mantle. Stolper and Newman (1994) estimated that addition of $0.1 \mathrm{wt} \% \mathrm{H}_{2} \mathrm{O}$ to the mantle source for the Mariana trough magmas resulted in about $6 \%$ increase in the degree of melting. Alternatively, Gallagher and Hawkesworth (1992) have suggested that in the presence of small amounts of water, the mantle lithosphere may yield basalt by decompression melting above an advancing plume. Such favorable conditions for lithospheric melting, however, are unlikely to persist (Arndt and Christiansen, 1992). Thus as rifting proceeds, primary magmatic inputs to the crust are expected to be dominated by asthenospheric-derived melt. The depleted character of the lavas at Site 917 may reflect melting of hydrated lithospheric mantle at the onset of volcanism, although this conjecture is speculative and needs rigorous testing.

\section{SUMMARY}

The results of melting experiments on primitive aphyric lavas from Site 917 define the phase relations governing crystal fractionation at low pressure for the continental volcanic succession. The li- 
quid lines of descent show close agreement with the compositions for primitive members of the Lower and Upper Series and the oceanic succession drilled at Sites 915 and 918, but contrast diverging trends for the more differentiated lavas from Site 917 . We propose that high$\mathrm{Al}_{2} \mathrm{O}_{3}$ differentiated lavas from the Lower Series were formed by an extended interval of olivine fractionation due to suppressed plagioclase crystallization at elevated $\mathrm{f}_{\mathrm{H} 2 \mathrm{O}}$. We also conclude from considerations of $\mathrm{Fe}-\mathrm{Mg}$ exchange equilibria for high-MgO aphyric lavas and picrites that most picrites have accumulated excess olivine and, therefore, do not represent liquid compositions. Some high-MgO aphyric flows, however, are permissible liquid compositions and may represent unfractionated mantle melts. Their major element compositions are in good agreement with primary liquid compositions calculated for aphyric lavas with $\sim 10 \mathrm{wt} \% \mathrm{MgO}$. The production of more $\mathrm{Fe}$ - and Ti-rich primary melts with time suggests that the efficiency of melt segregation and potential temperatures increased during production of the volcanic succession at Site 917. We emphasize that these conclusions have been reached from consideration of the phase relations and major element composition of Leg 152 lavas. These constraints on magma differentiation complement insights gained from considerations of trace elements (Fitton et al., Chap. 28, this volume; Larsen et al., Chap. 27, this volume; Fram et al., this volume) and from geophysical observations for the southeast Greenland volcanic rifted margin (Larsen, Saunders, Clift, et al., 1994).

\section{ACKNOWLEDGMENTS}

L.M. Larsen (Geological Survey of Greenland) kindly provided the XRF analyses of the starting materials and allowed us to use her database of whole rock analyses of the Leg 152 samples. Critical reviews by A.C. Kerr and A.D. Saunders were very helpful. Support for this research was provided by NSF-94-19382 (CEL) and a JOI/ USSAC grant to MSF.

\section{REFERENCES}

Arndt, N.T., and Christiansen, U., 1992. The role of lithospheric mantle in continental flood volcanism: thermal and geochemical constraints. $J$. Geophys. Res., 97:10967-10981.

Baker, D.R., and Eggler, D.H., 1983. Fractionation paths of Atka (Aleutians) high-alumina basalts: constraints from phase relations. J. Volcanol. Geotherm. Res., 18:387-404.

1987. Compositions of anhydrous and hydrous melts coexisting with plagioclase, augite, and olivine or low-Ca pyroxene from 1 atm to 8 kbar: applications to the Aleutian volcanic center of Atka. Am. Mineral., 72:12-28.

Beard, J.S., and Lofgren, G.E., 1992. An experiment-based model for the petrogenesis of high-alumina basalts. Science, 258:112-115.

Biggar, G.M., 1972. Diopside, lithium metasilicate and the 1968 temperature scale. Mineral. Mag., 38:768-770.

Blichert-Toft, J., Rosing, M.T., Lesher, C.E., and Chauvel, C., 1995. Geochemical constraints on the origin of the late Archean Skjoldungen alkaline igneous province, SE Greenland. J. Petrol., 36:515-561.

Bryan, W.B., Finger, L.W., and Chayes, F., 1969. Estimating proportions in petrographic mixing equations by least-squares approximation. Science, 163:926-927.

Fram, M.S., and Lesher, C.E., 1993. Geochemical constraints on mantle melting during creation of the North Atlantic basin. Nature, 363:712715 .

, 1997. Generation and polybaric differentiation of East Greenland Early Tertiary flood basalts. J. Petrol., 38:231-275.

Fram, M.S., and Longhi, J., 1992. Phase equilibria of dikes associated with Proterozoic anorthosite complexes. Am. Mineral., 77:605-616.

Gaetani, G.A., Grove, T.L., and Bryan, W.B., 1993. The influence of water on the petrogenesis of subduction-related igneous rocks. Nature, $365: 332-334$.

Gallagher, K., and Hawkesworth, C., 1992. Dehydration melting and the generation of continental flood basalts. Nature, 358:57-59.
Govindaraju, K., 1989. 1989 compilation of working values and sample description for 272 geostandards. Geostand. Newsl., 13 (spec. iss.):1113.

Grove, T.L., 1981. Use of FePt alloys to eliminate the iron loss problem in 1 atmosphere gas mixing experiments: theoretical and practical considerations. Contrib. Mineral. Petrol., 78:298-304.

Grove, T.L., and Kinzler, R.J., 1986. Petrogenesis of andesites. Annu. Rev. Earth Planet. Sci., 14:417-454.

Grove, T.L., Kinzler, R.J., and Bryan, W.B., 1992. Fractionation of midocean ridge basalt (MORB). In Morgan, J.P., Blackman, D.K., and Sinton, J.M. (Eds.), Mantle Flow and Melt Generation at Mid-Ocean Ridges. Geophys. Monogr., Am. Geophys. Union, 71:281-310.

Helz, R.T., and Thornber, C.R., 1987. Geothermometry of Kilauea Iki lava lake, Hawaii. Bull. Volcanol., 49:651-668.

Kilinc, A., Carmichael, I.S.E., Rivers, M.L., and Sack, R.O., 1983. The ferric-ferrous ratio of natural silicate liquids equilibrated in air. Contrib. Mineral. Petrol., 83:136-140.

Klein, E.M., and Langmuir, C.H., 1989. Local versus global variations in ocean ridge basalt composition: a reply. J. Geophys. Res., 94:4241-4252.

Kushiro, I., 1972. The effect of water on the composition of magmas formed at high pressures. J. Petrol., 13:311-334.

Langmuir, C.H., Klein, E., and Plank, T., 1992. Petrological systematics of mid-ocean ridge basalts: constraints on melt generation beneath ocean ridges. In Morgan, J., Blackman, D., Sinton, J. (Eds.), Mantle Flow and Melt Generation at Mid-Ocean Ridges. Geophys. Monogr., Am. Geophys. Union, 71:183-277.

Larsen, H.C., Saunders, A.D., Clift, P.D., et al., 1994. Proc. ODP, Init. Repts., 152: College Station, TX (Ocean Drilling Program).

Larsen, L.M., Watt, W.S., and Watt, M., 1989. Geology and petrology of the Lower Tertiary plateau basalts of the Scoresby Sund region, East Greenland. Bull.-Groenl. Geol. Unders., 157:1-164.

Longhi, J., 1991. Comparative liquidus equilibria of hypersthene-normative basalts at low pressure. Am. Mineral., 76:785-800.

Merzbacher, C., and Eggler, D.E., 1984. A magmatic geohygrometer: application to Mount St. Helens and other dacitic magmas. Geology, 12:587590.

Papike, J.J., Cameron, K.L., and Baldwin, K., 1974. Amphiboles and pyroxenes: characterization of other than quadrilateral components and estimates of ferric iron from microprobe data. Geol. Soc. Am. Abstr. Progr., 6:1053-1054. (Abstract)

Presnall, D.C., Dixon, S.A., Dixon, J.R., O’Donnell, T.H., Brenner, N.L., Schrock, R.L., and Dycus, D.W., 1978. Liquidus phase relations on the join diopside-forsterite-anorthite from 1 atm to 20 kbar: their bearing on the generation and crystallization of basaltic magma. Contrib. Mineral. Petrol., 66:203-220.

Roeder, P.L., and Emslie, R.F., 1970. Olivine-liquid equilibrium. Contrib. Mineral. Petrol., 29:275-289.

Sisson, T.W., and Grove, T.L., 1993. Experimental investigations of the role of $\mathrm{H}_{2} \mathrm{O}$ in calc-alkaline differentiation and subduction zone magmatism. Contrib. Mineral. Petrol., 113:143-166.

Spulber, S.D., and Rutherford, M.J., 1983. The origin of rhyolite and plagiogranite in oceanic crust: an experimental study. J. Petrol., 24:1-25.

Stolper, E., and Newman, S., 1994. The role of water in the petrogenesis of Mariana trough magmas. Earth Planet. Sci. Lett., 121:293-325.

Thompson, R.N., Esson, J., and Dunham, A.C., 1972. Major element chemical variation in the Eocene lavas of the Isle of Skye, Scotland. J. Petrol., 13:219-253.

Thy, P., 1995. Low-pressure experimental constraints on the evolution of komatiites. J. Petrol., 36:1529-1548.

Ulmer, P., 1989. The dependence of the $\mathrm{Fe}^{2+}-\mathrm{Mg}$ cations-partitioning between olivine and basaltic liquid on pressure, temperature and composition. An experimental study to 30 kbars. Contrib. Mineral. Petrol., 101:261-273.

Weaver, J.S., and Langmuir, C.H., 1990. Calculation of phase equilibrium in mineral-melt systems. Comput. Geosci., 16:1-19.

\footnotetext{
Date of initial receipt: 10 November 1995

Date of acceptance: 21 May 1996

Ms 152SR-235
} 